\title{
Type-2 Fuzzy Elliptic Membership Functions for Modeling Uncertainty
}

\author{
Erdal Kayacan ${ }^{\mathrm{a}}$, Andriy Sarabakha ${ }^{\mathrm{a}}$, Simon Coupland ${ }^{\mathrm{b}}$, Robert John ${ }^{\mathrm{c}}$, Mojtaba \\ Ahmadieh Khanesar ${ }^{\mathrm{d}}$ \\ ${ }^{a}$ School of Mechanical and Aerospace Engineering, Nanyang Technological University, 639798, \\ Singapore. e-mail: erdal@ntu.edu.sg, andriy001@e.ntu.edu.sg \\ ${ }^{b}$ School of Computer Science and Informatics, De Montfort University, The Gateway, Leicester, \\ LE1 9BH, UK. e-mail: s imonc@ dmu . ac . uk \\ ${ }^{c}$ The Lab for Uncertainty in Data and Decision Making (LUCID), School of Computer Science, \\ University of Nottingham, Nottingham, NG8 1BB, UK. e-mail: \\ Robert. John@ nottingham. ac.uk \\ ${ }^{d}$ DTU Compute, Denmark, Asmussens All Building 303B, 2800 Kgs. Lyngby \\ e-mail: makh@dtu.dk
}

\begin{abstract}
Whereas type-1 and type-2 membership functions (MFs) are the core of any fuzzy logic system, there are no performance criteria available to evaluate the goodness or correctness of the fuzzy MFs. In this paper, we make extensive analysis in terms of the capability of type-2 elliptic fuzzy MFs in modeling uncertainty. Having decoupled parameters for its support and width, elliptic MFs are unique amongst existing type-2 fuzzy MFs. In this investigation, the uncertainty distribution along the elliptic MF support is studied, and a detailed analysis is given to compare and contrast its performance with existing type-2 fuzzy MFs. Furthermore, fuzzy arithmetic operations are also investigated, and our finding is that the elliptic MF has similar features to the Gaussian and triangular MFs in addition and multiplication operations. Moreover, we have tested the prediction capability of elliptic MFs using interval type-2 fuzzy logic systems on oil price prediction problem for a data set from 2nd Jan 1985 till 25th April 2016. Throughout the simulation studies, an extreme learning machine is used to train the interval type- 2 fuzzy logic system. The prediction results show that, in addition to their various advantages mentioned above, elliptic MFs have comparable prediction results when compared to Gaussian and triangular MFs. Finally, in order to test the performance of fuzzy logic controller with elliptic interval type-2 MFs, extensive real-time experiments are conducted for the $3 \mathrm{D}$ trajectory tracking problem of a quadrotor. We believe
\end{abstract}


that the results of this study will open the doors to elliptic MFs' wider use of real-world identification and control applications as the proposed MF is easy to interpret in addition to its unique features.

Keywords: Elliptic membership function, type-2 fuzzy logic theory, uncertainty, fuzzy sets, Gaussian, triangular, addition, multiplication, fuzzy arithmetics.

\section{Introduction}

Fuzzy logic has had a significant impact on identification and control problems since it was firstly proposed by Zadeh in 1965 [1]. Fuzzy logic owes its exceptional scientific reputation to its unique ability to simultaneously deal with uncertainties in the system and use the expert knowledge as an input to the fuzzy system design. As an extension of type-1 fuzzy logic systems (T1FLSs), their type-2 counterparts - type-2 fuzzy logic systems (T2FLSs) - have also made an impact on dealing with uncertainties over the last two decades. The concept of type-2 fuzzy sets first appeared in 1975 [2]. Unfortunately, researchers had to wait for a while the theory to be developed more by Mendel and Karnik [3]. The progress of T2FLSs was primarily impeded by algorithmic and hardware limitations. Whereas the former refers to the procedure of starting from the type- 2 fuzzy set and ending up with a crisp number which is called a sequence of two operations: type reduction and defuzzification, the latter refers to the relatively low computational power of processors. In particular, type reduction is challenging, because no closed form formulation exists for type-reduction as the only option is to use iterative algorithms such as Karnik-Mendel algorithm. These limitations delayed the real-time implementations of T2FLSs until the 1990s. Luckily, these limitations have diminished over the time due to several simplified algorithms which make the type reduction operation easier and relatively simpler, especially those who do not need iterative algorithms.

Since the working principle of fuzzy logic is similar to the human way of thinking, the fuzzy logic theory has been extensively implemented in function approximation and control systems. In particular, in real-time control applications, there is a significant amount of uncertainty inherent in the system due to, interalia, convoluted nonlinear dynamics in the actuators, noise from both internal and external sources, lack of modeling, changing working conditions as well as requirements for guaranteed stability and satisfactory performance of the system with a wide range of conditions. Intelligent, or model-free, control methodologies can address such a broad scope of challenges simultaneously, and amongst such 
methods, fuzzy control is one of the most successful techniques.

The theoretical basis of the interval T2FLSs, simplified version of generalized T2FLSs, was described in [4]. While the third dimension in the footprint of uncertainty (FOU) of a generalized T2FLS may have an arbitrary number resulting in a non-smooth 3D geometry, the third dimension is always equal to one in an interval T2FLS. Generalized T2FLSs, in particular, the type reduction operation, are indisputably more difficult when compared to their interval counterparts. A detailed tutorial for generalized T2FLSs can be seen in [5].

As a further simplification to T2FLSs, many type reduction algorithms have also been proposed including those does not need any type reduction phase. In turn, such simplifications and algorithmic revolutions have opened the doors of type-2 fuzzy logic controller real-time implementations within the spheres of mobile robotics, mechatronics [6], aerospace [7, 8, 9, 10] and any other control theory applications. The transition from T1FLS to T2FLS is still a research topic as T2FLSs appear to be a promising approach for handling uncertainties in realworld applications, which exhibit measurement noise and any other types of uncertainties [11, 12, 13]. The use of the T2FLSs has the potential to provide better performance when compared to a T1FLS [14]. This claim does not mean that such a potential will result in a better performance every time. A high-level retrospective of T2FLSs and their type-1 counterparts can be seen in [15].

Interval T2FLSs have tremendous potential to be used in identification, control, classification, time series prediction, signal processing, image processing and decision making systems, which have both internal and external uncertainties, by virtue of their following discriminating factors including, inter-alia:

- More degrees of freedom: Similar to the fact that T1FLSs have more degrees of freedom than the crisp systems, T2FLSs have more degrees of freedom from their design point of view.

- Power of FOU: There is no need to place the membership functions (MFs) precisely as there is an infinite number of type- 1 MFs in the FOU of a T2FLS.

- Handling uncertainty: The noise rejection capability of T2FLSs is better than T1FLSs

- Representation of uncertainty: T2FLSs are more convenient for representing uncertainty when compared to T1FLSs 
In literature, the items above are sometimes misinterpreted in a way that a T2FLS must give a more optimal response when compared to its type-1 counterpart. This is a serious paralogism because a number of papers have claimed that T2FLSs give a significant improvement when there exists a significant amount of uncertainty in the system. This conclusion can be summarized and simplified as: T2FLSs must be used when needed. A more generalized analysis can be found in [16].

MFs were introduced by Zadeh in his paper on fuzzy sets in 1965. The basic definitions of fuzzy logic and MFs of Zadeh were as follows:

"A fuzzy set is a class of objects with a continuum of grades of membership. Such a set is characterized by a membership (characteristic) function which assigns to each object a grade of membership ranging between zero and one." [1].

L. A. Zadeh

In the type-2 fuzzy logic research community, we characterise type-2 fuzzy MFs by the shape of the FOU. There exist a number of type- 2 fuzzy MFs in literature, (i.e. triangular, trapezoidal, Gaussian, two-sided Gaussian, Bell-shaped, sigmoid, pi-shaped). However, all the aforementioned MFs have an adverse common feature: the parameters responsible for the support and the width are coupled. By the use of elliptic MF [17], the parameters responsible for the width of uncertainty are de-coupled from the parameters responsible for the center and the support of the MF. This feature allows us to analyze how the uncertainty in the input distorts the inference of the T2FLS [16]. Another attempt in the literature to describe a similar MF was disjunctive strictly-monotonic and conjunctive nonstrictly monotonous MFs in which the user has to determine four parameters [18]. However, the mathematical formulation defined in this attempt is too complicated to be used in any engineering application.

Even if there are some fuzzy logic software packages which allow the user to design custom shaped MFs, there is no systematic way to choose a fuzzy MF in order to achieve a better control performance. Surprisingly, there is a relatively small literature about choosing an appropriate MFs in a T2FLS. Several membership generation methods are investigated in [19], and the overall finding is that there is no single best method; the choice of the method depends on the particular problem. However, this work has specialized only for pattern recognition applications. Another study has investigated the effect of type- 2 fuzzy MFs on modeling variation in human decision making, [20] and it has been shown that there is a direct relationship between the variation in decision making and the uncertainty introduced to the membership functions. 
This paper aims to fill this gap by elaborating on the similarities and contrasts between the conventional type-2 fuzzy MFs and elliptic MFs. In a recent work [21], authors have investigated a special version of elliptic MFs, which are semielliptic MFs. In this paper, we further extend the analysis to general elliptic MFs. In our analysis, we look at the uncertainty modeling capability of different type-2 MFs and make some comments about fuzzy arithmetic, by using the novel elliptic MF.

Since elliptic MFs have firstly been introduced, their several simulation and real-time implementations have already been published [22]. However, the shape of the novel MF and its features have never been elaborated. The novelties of this paper are:

- Since the points, at which the MF is zero and one, are certain, the consequent part parameters can be set using the values at exactly the center of the MFs for function approximation implementations.

- From the linguistic point of view, if we are certain about what belongs to a set and what does not, we can use elliptic MFs.

- Uncertainty measures for the elliptic MF is done for the first time, and the results are compared with that of the triangular MF.

- Arithmetic operations in elliptic MFs do not change its shape.

Paper Organization: The rest of the paper is organized as follows: In Section 2, we go over both the existing type-2 MFs and elliptic MF. In Section 3, we give a brief critique of their capability of modeling uncertainty. In Section 4, function approximation capability of the fuzzy system using elliptic type- $2 \mathrm{MF}$ is investigated. Uncertainty modeling capability of the type- 2 fuzzy system with elliptic type-2 MF is investigated in Section 5. Function approximation capability of the type-2 fuzzy system using elliptic type-2 MF is investigated in Section 6. Section 7 presents fuzzy arithmetics by using different type-2 MFs. In Section 8, the capability of elliptic interval type-2 MFs to predict the oil price is tested. In Section 9, real-time experimental tests are conducted for the 3D trajectory tracking problem of a quadrotor unmanned aerial vehicle, in order to validate the control performances of elliptic type-2 MFs. Finally, some conclusions are drawn from this study in Section 10. 


\section{Type-2 Fuzzy Logic Systems}

A type-2 fuzzy set is where the membership grade is not in $[0,1]$ but is itself a (type-1) fuzzy set. Zadeh introduced the notion of type-n fuzzy sets.

Definition: Type-n fuzzy sets [2]. A fuzzy set is of type $n, n=2,3, \ldots$, if its $M F$ ranges over fuzzy sets of type $n-l$. The MF of a fuzzy set of type 1 ranges over the interval $[0,1]$.

This elegant definition provides an excellent, concise description of type-n fuzzy sets. It is worth noting here that, to date, researchers are working with type-1 and type-2 fuzzy sets with limited research into type-n fuzzy sets [23].

Now we introduce a more modern definition:

Definition: A Type-2 Fuzzy Set [24] is a bivariate function on the cartesian product $X \times[0,1]$ into $[0,1]$. In other words, $\mu$ on the universe of discourse $X$ is given by $u: X \times[0,1] \rightarrow[0,1]$.

This paper is concerned with interval type-2 fuzzy sets.

Definition: An interval type-2 fuzzy set is where the type-1 fuzzy set in the secondary takes value unity everywhere. That is an Interval type-2 fuzzy set is a function from $X$ into $D[0,1]$ where $D[0,1]$ is the set of closed sub-intervals in $[0,1]$ [24].

An interval type-2 fuzzy set is fully defined by it's FOU.

Definition [24]: The FOU is the union of all the primary memberships and is given by $\{(x, t) \in X \times[0,1] \mid \mu(x, t)>0\}$. As stated in [24] it is the support of $\mu$.

\section{Transition From Type-1 to Type-2 and Existing Type-2 MFs in the Litera- ture}

All the existing type-2 fuzzy MFs in the literature are nothing but the modified versions of the conventional type- 1 fuzzy MFs. In other words, the basis, the original Gaussian type-1 fuzzy MF in Fig. 1, is blurred once the expert is not sure about the membership values around a certain point over the support. The transition from type-1 to type- 2 is clearly seen in Fig. 1 .

In Fig. 1, the expert is pretty sure about the membership values around the left and right endpoints over the support. On the other hand, there is ambiguity for the expert for the membership values around the center of the MF which is 3 on the support.

Although there are a number of fuzzy MFs in the literature, two of them are commonly preferred: Gaussian and triangular type-2 fuzzy MFs. While the former has an advantage of being differentiable along the support which is advantageous especially when designing parameter update rules for adaptive neuro-fuzzy 


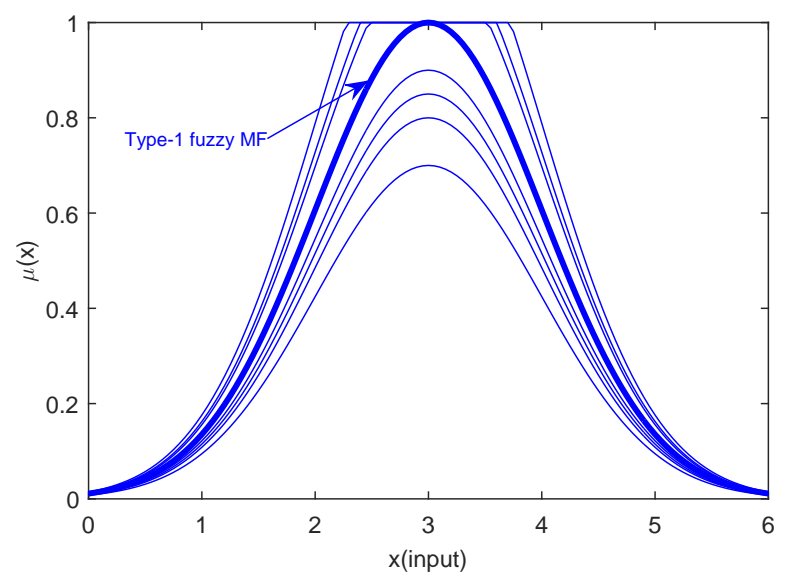

Figure 1: Transition from type-1 to type-2 fuzzy MF.

systems [25], the latter has linear regions which may make some analysis attempts easier. What is more, Gaussian MFs are always continuous which is not always the case for some other MFs.

Unlike Fig. 1 where the center of the Gaussian MF is blurred, another possibility is that the standard deviation of the MF may also be blurred. In Figs. 2(a) and 2(b), Gaussian type-2 fuzzy sets with uncertain standard deviation and uncertain mean are shown, respectively. The mathematical expression for the Gaussian MF is expressed as:

$$
\tilde{\mu}(x)=\exp \left(-\frac{1}{2} \frac{(x-c)^{2}}{\sigma^{2}}\right)
$$

where $c$ and $\sigma$ are the center and the width of the MF, $x$ is the input vector.

In Figs. 2(c) and 2(d), triangular type-2 fuzzy sets with uncertain width and uncertain center are shown. The mathematical expression for the MF is expressed as:

$$
\tilde{\mu}(x)=\left\{\begin{array}{cc}
1-\frac{|x-c|}{d} & \text { if } c-d<x<c+d \\
0 & \text { else }
\end{array},\right.
$$

where $c$ and $d$ are the center and the width of the MF, $x$ is the input vector.

In almost all the applications of fuzzy logic theory, MFs are chosen based on subjective perceptions of vague or imprecise categories rather than on data or other objective entities involved in the given problem [26]. Moreover, there are no performance criteria available to evaluate the goodness or correctness of the 


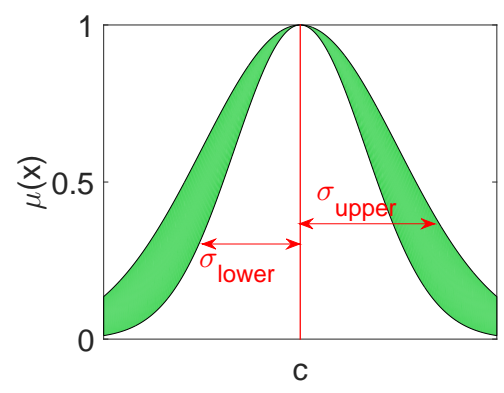

(a) uncertain standard deviation

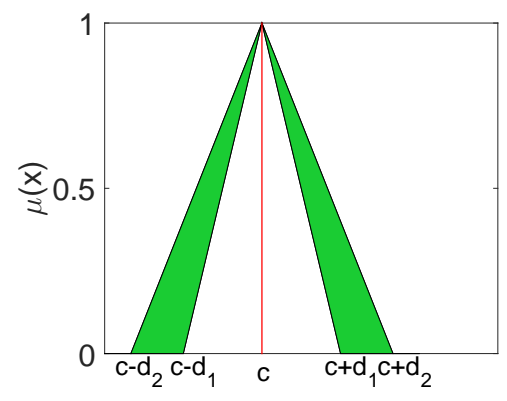

(c) uncertain width

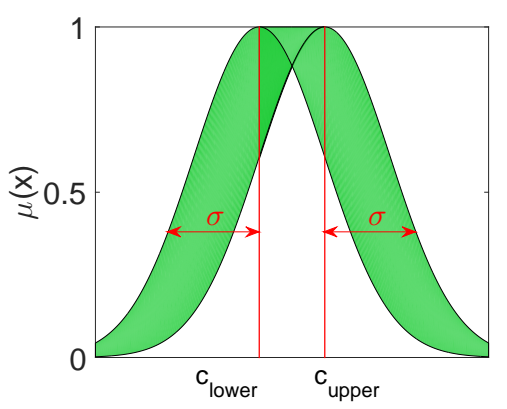

(b) uncertain mean

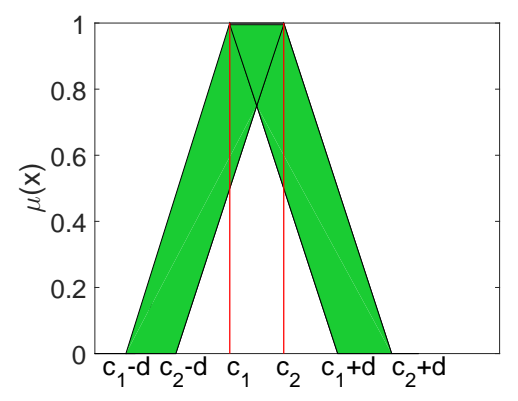

(d) uncertain center

Figure 2: Type-2 fuzzy Gaussian and triangular MFs.

fuzzy MFs [26]. There are some attempts to which type-2 MFs are the best for which application. For instance, in [27], a detailed analysis has been done on Gaussian and trapezoidal MFs in interval type-2 fuzzy logic controllers. It has been reported that interval type- 2 fuzzy logic controllers by using Gaussian MFs are simpler in design, always continuous and faster for small rule-bases, whereas the controllers by using trapezoidal MFs are simpler in analysis [27]. Another path in research is the auto-generation of fuzzy MFs [28], however, the interpretation of these methods is still limited.

Since the main motivation of this investigation is to analyse the elliptic MFs [16], we concentrate on them in this subsection. Elliptic MFs have certain values on the left and right end of the support, and it deals with uncertainty on the rest of the support. Inspired by the formulation of the triangular MFs, the lower and the upper MFs are defined with the following formulas:

$$
\bar{\mu}(x)=\left\{\begin{array}{cc}
\left(1-\left|\frac{x-c}{d}\right|^{a_{1}}\right)^{1 / a_{1}} & \text { if } c-d<x<c+d \\
0 & \text { else }
\end{array}\right.
$$




$$
\underline{\mu}(x)=\left\{\begin{array}{cc}
\left(1-\left|\frac{x-c}{d}\right|^{a_{2}}\right)^{1 / a_{2}} & \text { if } c-d<x<c+d \\
0 & \text { else }
\end{array}\right.
$$

where $c$ and $d$ are the center and the width of the MF, $x$ is the input vector. The parameters $a_{1}$ and $a_{2}$ determine the width of the uncertainty of the proposed MF, and these parameters should be selected in the following form:

$$
\begin{array}{r}
a_{1}>1 \\
0<a_{2}<1 .
\end{array}
$$

Figures 3(a), 3(b), 3(c) and 3(d) show the shapes of the proposed MF for $a_{1}=a_{2}=1, a_{1}=1.2, a_{2}=0.8, a_{1}=1.4, a_{2}=0.6$ and $a_{1}=1.6, a_{2}=0.4$, respectively. As can be seen from Fig. 3(a), the shape of the proposed type$2 \mathrm{MF}$ is changed to a type- 1 triangular MF when its parameters are selected as $a_{1}=a_{2}=1$.

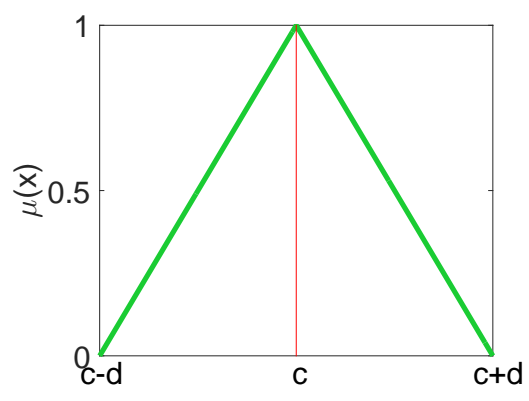

(a) $a_{1}=1$ and $a_{2}=2$

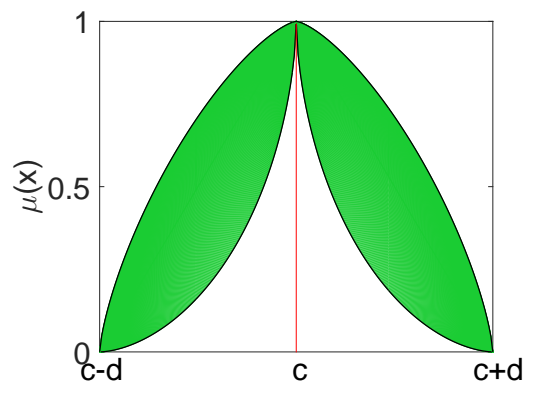

(c) $a_{1}=1.4$ and $a_{2}=0.6$

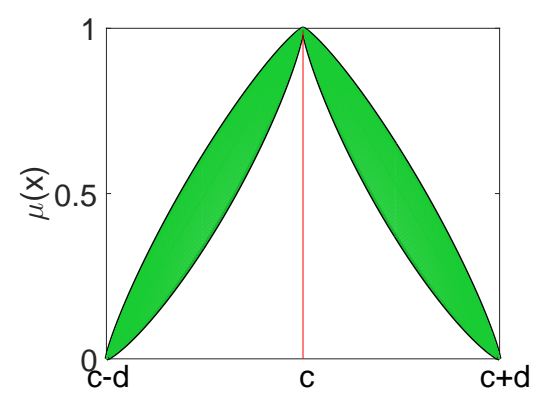

(b) $a_{1}=1.2$ and $a_{2}=0.8$

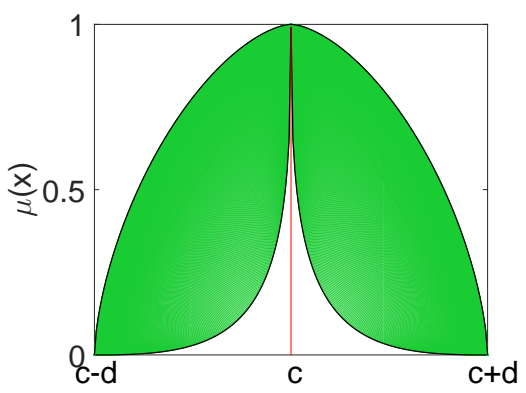

(d) $a_{1}=1.6$ and $a_{2}=0.4$

Figure 3: The shape of the proposed type-2 MF for different $a_{1}$ and $a_{2}$. 
The elliptic type-2 fuzzy MF has already been used for identification [29] and control [22] purposes, and performed promising results.

The diamond-shaped type-2 fuzzy MFs, which have similar features to elliptic MFs, were also proposed [30]. Their similarities to elliptic MFs are to have certain values both on the left and right end-points of the support and to represent uncertainty on the rest of the support. However, elliptic MFs are smoother than the diamond-shaped type-2 fuzzy MFs and are differentiable in more points of its support set than diamond-shaped type-2 MF. Moreover, the diamond-type type-2 MFs have more parameters to tune than elliptic type-2 MF which make it hard to tune.

\section{Function Approximation Using Different Fuzzy MFs}

\subsection{Function approximation using triangular type-1 MFs}

The design procedure of a type- 1 fuzzy function for the approximation of the function $g(x)$ is reviewed in this subsection [31].

- Define $N_{I}(i=1,2)$ fuzzy sets $A_{i}^{1}, A_{i}^{2}, \ldots, A_{i}^{N_{i}}$ in the support set of the system defined by $\left[\alpha_{i}, \beta_{i}\right]$. The MFs considered are triangular type-1 MFs which are considered to be normal, consistent and complete as follows:

$$
\mu_{A_{i}^{j}}\left(x_{i}\right)=\mu_{A_{i}^{j}}\left(x_{i}, e_{i}^{j-1}, e_{i}^{j}, e_{i}^{j+1}\right),
$$

where:

$$
\alpha_{i}=e_{i}^{1}<e_{i}^{2}<\ldots<e_{i}^{N_{i}}=\beta_{i} .
$$

- Construct $M=N_{1} \times N_{2}$ fuzzy $I F-T H E N$ rules in the following form:

$$
\text { If } x_{1} \text { is } A_{1}^{i_{1}} \text { and } x_{2} \text { is } A_{2}^{i_{2}} \text { Then } y \text { is } B^{i_{1} i_{2}} \text {, }
$$

where $i_{1}=1, \ldots, N_{1}, i_{2}=1,2, \ldots, N_{2}$ and the center of the fuzzy sets are $B^{i_{1} i_{2}}$ denoted by $\bar{y}^{i_{1} i_{2}}$ is chosen as:

$$
\bar{y}^{i_{1} i_{2}}=g\left(e_{1}^{i_{1}}, e^{i_{2}}\right)
$$

- Construct the fuzzy system $f(x)$ using the product inference engine, singleton fuzzifier and center average defuzzifier as follows:

$$
f(x)=\frac{\sum_{i_{1}=1}^{N_{1}} \sum_{i_{2}=1}^{N_{2}} \bar{y}^{i_{1} i_{2}}\left(\mu_{A_{1}^{i_{1}}}\left(x_{1}\right) \mu_{A_{2}^{i_{2}}}\left(x_{2}\right)\right)}{\sum_{i_{1}=1}^{N_{1}} \sum_{i_{2}=1}^{N_{2}}\left(\mu_{A_{1}^{i_{1}}}\left(x_{1}\right) \mu_{A_{2}^{i_{2}}}\left(x_{2}\right)\right)} .
$$


Theorem. Let $f(x)$ be the fuzzy system designed through the above three steps. If the $g(x)$ is twice continuously differentiable on $U$, then [31]:

$$
\|g-f\|_{\infty}=\frac{1}{8}\left[\left\|\frac{\partial^{2} g}{\partial x_{1}^{2}}\right\|_{\infty} h_{1}^{2}+\left\|\frac{\partial^{2} g}{\partial x_{2}^{2}}\right\|_{\infty} h_{2}^{2}\right],
$$

where $h_{i}=\max _{1 \leq j \leq N_{i}-1}\left|e_{i}^{j+1}-e_{i}^{j}\right|, i=1,2$

\subsection{Function approximation using elliptic type-2 $M F$}

As can be seen from the algorithm considered in the previous subsection, it is possible to use triangular type-1 MFs to approximate a nonlinear function. The firing of triangular type-1 MF used are equal to one where the firing of the next MF is equal to zero, this is the key feature to determine the value of the corresponding values of the consequent part parameters.

Since the exact value of a function is known when the input to a fuzzy system is exactly equal to the center value, it is required for a type- 2 MF to be exactly equal to one in the center and be exactly equal to zero at both ends of the support set. The only type- 2 MF which benefits from this feature is elliptic type- $2 \mathrm{MF}$. In other words, if for example a triangular type-2 MF which has uncertain values at center and/or its values at its both ends are uncertain, it is too difficult to determine the exact values of its consequent part parameters.

\section{Uncertainty Modeling Capability}

A gigantic amount of publications in fuzzy logic theory, no doubt almost all of them report the superiority of fuzzy logic systems over the conventional methods, may cause the readers get lost in this big ocean; and frankly speaking, only a few of those concentrate on the meaning of uncertainty while their basic claim is to be able to deal with it. In this section, we make an attempt to consider all these sets to form the perspective of uncertainty modeling. This is, by its very nature, somewhat subjective but we hope is interesting for the reader.

\subsection{Uncertainty distribution along the support}

The capability of modeling uncertainty plays a vital role not only in control systems but also in decision support systems. In particular, fuzzy decision-making systems are designed in a way that they will be able to model the uncertainty resulting from a strong and accurate decision support system. Unfortunately, there exist neither a systematic way to choose a fuzzy MF in order to achieve a better uncertainty modeling capability nor an objective criterion to check its performance. 
We aim to make another subjective analysis in this section in which we will compare and contrast the different ways of modeling uncertainty for different type-2 MFs in literature.

\subsubsection{Gaussian and triangular $M F S$}

In both Gaussian and triangular type-2 fuzzy MFs, the FOU is usually created in two ways: either blurring the width (or standard deviation) of the MF around a fixed center or blurring the center of the MF around fixed width (or standard deviation). While the former refers to Figs. 2(a) and 2(c), the latter refers to Figs. 2(b) and 2(d). According to our analysis, none of them results in a cogent argument from the uncertainty modeling capability point of view.

First, let us consider Figs. 2(a) and 2(c). For both figures, let us imagine a horizontal line over the MFs. When we place the horizontal line on $\mu(x)=1$ (it will result in a tangential line to the MF), then our comment would be that the expert is pretty sure about the location of the MF around the center which means uncertainty is less around the center of the MF. The reason is that for a specific membership value, which is $\mu(x)=1$, there is only one input which is the center. In other words, there is no uncertainty on this line. On the other hand, when we move the line downwards, for a specific membership value, there will be wider input regions which means that the expert is more uncertain when the inputs become distant from the center. When we continue moving the horizontal line more downwards, for a specific membership value, for instance when $\mu(x)$ is very low, the input region will be the widest which means that the expert is very uncertain. The former part may be reasonable as the expert is probably more precise around the center of the MF, however, it is being more ambiguous along the support as being far away from the center. The latter part is not realistic from a human expert point of view because if an expert is precise around the center that a specific input belongs to a fuzzy set, the same expert must be also precise that after some distance along the support, the input will definitely not belong to this specific set. In this case, it is not reasonable to increase the uncertainty constantly. Therefore, this type of representation is not convincing. Second, let us consider Figs. 2(b) and 2(d), For both figures, let us imagine a horizontal line over the MFs. We can easily realize that for a specific membership value, the input range is always the same even if we move the line downwards. In other words, the uncertainty is always equal along the support. This type of uncertainty modeling is also not reasonable as the expert cannot be equally uncertain along the support. As he/she is the person who places the MF on a specific center value, we expect $\mathrm{him} /$ her to be more or less uncertain on the specific places over the support. 


\subsubsection{Elliptic $M F s$}

In this subsection, we will show that when a similar analysis in the previous subsection is carried out for the elliptic type-2 fuzzy MFs, different conclusions are reached. Let us consider Figs. 3(b) and 3(c) where the expert is very precise that a specific input around the center belongs to a specific fuzzy set. The reason is that a horizontal line is drawn on $\mu(x)=1$ (it will result in a tangential line to the MF), then our reasonable comment would be that the expert is pretty sure about the location of the MF around the center which means uncertainty is less around the center of the MF. This is something that we expect from an expert that he/she is the person who chooses the centers of the MFs. What is more, as we move the horizontal line downwards, in other words moving from the center towards the left or right end-points of the support $(c+d$ or $c-d)$, the expert is not certain anymore about his/her decision. It is subtle that after the halfway over its support, the expert starts being more precise that a specific input does not belong this fuzzy set. The reason for such a modeling is explained in the next paragraph with the help of Fig. 4 .

In order to be able to make our analysis in a more clear way, let's consider a case where we have more than one MF which is illustrated in Fig. 4. Whereas the first elliptic type- 2 fuzzy MF is centered at 0 , the other is centered at 1 . The expert is quite precise that the first input, 0.1, belongs to the magenta fuzzy set. However, the expert is again precise that the third input, 0.9 , does not belong to the magenta fuzzy set. On the other hand, the expert is again precise that the third input, 0.9, belongs to the green fuzzy set whereas he/she is again precise that the first input, 0.1 , does not belong to the green fuzzy set. The expert is most uncertain around 0.5 . We believe that this way of uncertainty representation is closer to the human way of thinking when compared to Gaussian or triangular MFs.

\section{The Comparison Between the Uncertainty Measures of Elliptic Type-2 MF with Triangular One}

In this section, in order to compare the entropy of the elliptic type- $2 \mathrm{MF}$ with the triangular one, some experiments are performed. These tests are based on uncertainty measures for interval type- 2 fuzzy sets which are studied by $\mathrm{Wu}$ and Mendel [32].

A type-1 fuzzy set is said to be completely fuzzy if its degrees of membership are always equal to 0.5 in all the points in its support set. For a general type- 1 fuzzy, the degrees of fuzziness is defined based on their similarity with the type- 1 fuzzy set with complete fuzziness. There exist different fuzziness measures for 


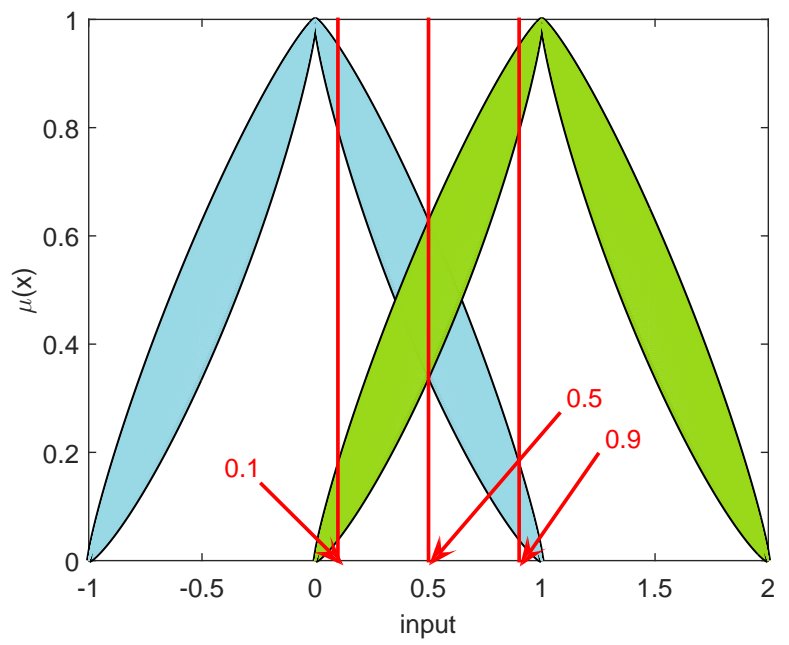

Figure 4: Having more than one elliptic MF.

type-1 fuzzy sets which are extended to interval type-2 fuzzy MFs. There are different uncertainty measures in literature; while the results obtained by these uncertainty measures can be whether crisp numbers or interval values. Two uncertainty measures which result in a crisp number are as follows:

1. The uncertainty measure proposed by Szmidt and Kacprzyk [33]:

$$
F_{S K}(\tilde{A})=\frac{1}{N} \sum_{i=1}^{N} \frac{1-\max \left[1-\bar{\mu}_{\tilde{A}}\left(x_{i}\right), \underline{\mu}_{\tilde{A}}\left(x_{i}\right)\right]}{1-\min \left[1-\bar{\mu}_{\tilde{A}}\left(x_{i}\right), \underline{\mu}_{\tilde{A}}\left(x_{i}\right)\right]} .
$$

2. The uncertainty measure proposed by Zeng and Li [34]:

$$
F_{Z L}(\tilde{A})=\frac{1}{N} \sum_{i=1}^{N}\left|\bar{\mu}_{\tilde{A}}\left(x_{i}\right)+\underline{\mu}_{\tilde{A}}\left(x_{i}\right)-1\right| .
$$

Two uncertainty measures which result in an interval set are as follows:

1. The uncertainty measure proposed by Cornelis and Kerre [35]:

$$
\begin{aligned}
F_{Z L}(\tilde{A})= & {\left[\frac{2}{N} \sum_{i=1}^{N} \min \left(\underline{\mu}_{\tilde{A}}\left(x_{i}\right), 1-\bar{\mu}_{\tilde{A}}\left(x_{i}\right)\right),\right.} \\
& \left.\frac{2}{N} \min \left(0.5,1-\underline{\mu}_{\tilde{A}}\left(x_{i}\right), \bar{\mu}_{\tilde{A}}\left(x_{i}\right)\right)\right] .
\end{aligned}
$$


2. The definition of fuzziness as an interval based on the Mendel-John Representation Theorem [36].

$$
F_{M J}(\tilde{A})=\left[f_{l}(\tilde{A}), f_{r}(\tilde{A})\right] .
$$

This interval can be calculated using the theorem given by $\mathrm{Wu}$ and Mendel [32] as follows: Let $A_{e 1}$ be defined as:

$$
\mu_{A e 1}= \begin{cases}\bar{\mu}_{\tilde{A}}\left(x_{i}\right) & \bar{\mu}_{\tilde{A}}\left(x_{i}\right) \text { is further away from } \\ & 0.5 \text { than } \underline{\mu}_{\tilde{A}}\left(x_{i}\right) \\ \underline{\mu}_{\tilde{A}}\left(x_{i}\right) & \text { otherwise, }\end{cases}
$$

and $A_{e 2}$ is defined as:

$$
\mu_{A e 2}=\left\{\begin{aligned}
\bar{\mu}_{\tilde{A}}\left(x_{i}\right) & \text { if both } \bar{\mu}_{\tilde{A}}\left(x_{i}\right) \text { and } \underline{\mu}_{\tilde{A}}\left(x_{i}\right) \text { are below } 0.5 \\
\underline{\mu}_{\tilde{A}}\left(x_{i}\right) & \text { if both } \bar{\mu}_{\tilde{A}}\left(x_{i}\right) \text { and } \underline{\mu}_{\tilde{A}}\left(x_{i}\right) \text { are above } 0.5 \\
0.5 & \text { otherwise. }
\end{aligned}\right.
$$

Then:

$$
\begin{aligned}
& f_{l}(\tilde{A})=f\left(A_{e 1}\right) \\
& f_{r}(\tilde{A})=f\left(A_{e 2}\right),
\end{aligned}
$$

where $f($.$) is any of type- 1$ fuzziness measures. In this study, as it is also suggested by $\mathrm{Wu}$ and Mendel [37], the fuzziness measure proposed by Yager is preferred as follows:

$$
f_{Y}(A)=1-\frac{\left[\sum_{i=1}^{N}\left|2 \mu_{A}\left(x_{i}\right)-1\right|^{r}\right]^{1 / r}}{N^{1 / r}} .
$$

The equation used for a triangular type-2 MF is as follows:

$$
\begin{gathered}
\underline{\mu}= \begin{cases}0 & x<t-1 \\
x+1-t & t-1<x<0 \\
1-x-t & 0<x<1-t \\
0 & \text { otherwise }\end{cases} \\
\bar{\mu}= \begin{cases}0 & x<-1 \\
\frac{x+1}{1-t} & -1<x<-t \\
1 & -t<x<t \\
\frac{x-1}{t-1} & t<x<1 \\
0 & \text { otherwise, }\end{cases}
\end{gathered}
$$


where $t$ is a positive value from the interval of $[0,1]$. In order to have a fair comparison between the fuzziness of triangular type- $2 \mathrm{MF}$ and elliptic one, the area of the FOU of the two interval type-2 fuzzy sets are kept the same. The area of the FOU for triangular type-2 MF is calculated as follows:

$$
\text { Area }=(1+t)-(1-t)^{2} \text {. }
$$

In order to obtain the area of the FOU of a type- 2 MF whose parameters are selected as $c=0, d=1$ and $a_{1}=a_{2}^{-1}=a$, the following $\beta$ function is used:

$$
\beta(z, w)=\int_{0}^{1} t^{z-1}(1-t)^{w-1} d t=\frac{\Gamma(z) \Gamma(w)}{\Gamma(z+w)} .
$$

The area of FOU for elliptic type-2 MF is obtained as follows:

$$
I=\int_{-1}^{1}\left(1-|x|^{a}\right)^{1 / a}-\left(1-|x|^{a}\right)^{1 / a} .
$$

This area can be calculated using the $\beta$ function as follows:

$$
I=2 \frac{\beta\left(a^{-1}+1, a^{-1}\right)}{a}-2 a \beta(a+1, a) .
$$

In order to make the area of FOU of both type-2 MFs the same, the parameter $t$ of triangular type- $2 \mathrm{MF}$ is incremented from 0 by 0.01 to 0.5 . The area of triangular type- $2 \mathrm{MF}$ is calculated and solving the following equation:

$$
I=2 \frac{\beta\left(a^{-1}+1, a^{-1}\right)}{a}-2 a \beta(a+1, a)=(1+t)-(1-t)^{2}
$$

the appropriate value of $a$ is found. It is then possible to achieve the fuzziness measures using each of aforementioned formulas for calculating uncertainty measure.

The result of applying the uncertainty measure proposed by Szmidt and Kacpryzk and the one proposed by Zeng and Li are depicted in Figs 5(a) and 5(b), respectively. As can be seen from these figures, the uncertainty measure for the elliptic type-2 MF is larger than that of triangular type-2 MF. Two interval valued uncertainty measures based on Cornelis and Kerre uncertainty measure and MendelJohn Representation theorem are also depicted in Figs 5(c) and 5(d), In these figures, the interval representing the fuzziness of elliptic type-2 MF is colored blue while that of triangular type-2 MF is yellow. There is a common interval between these two intervals which is shown by green which is the mixture of yellow and blue. As can be seen from these figures, the lower value of the interval and its upper value are both larger for elliptic type-2 MF are larger than that of triangular type-2 MF. 


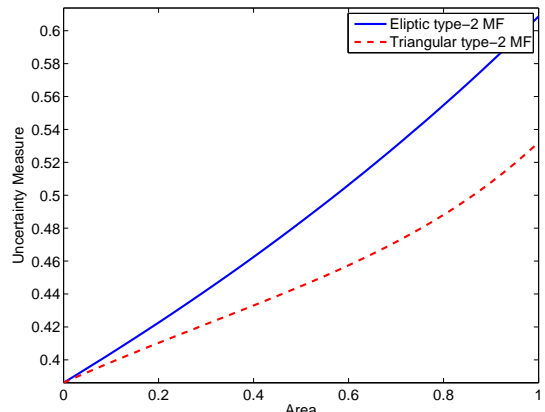

(a) Method proposed by Szmidt and Kacpryzk.

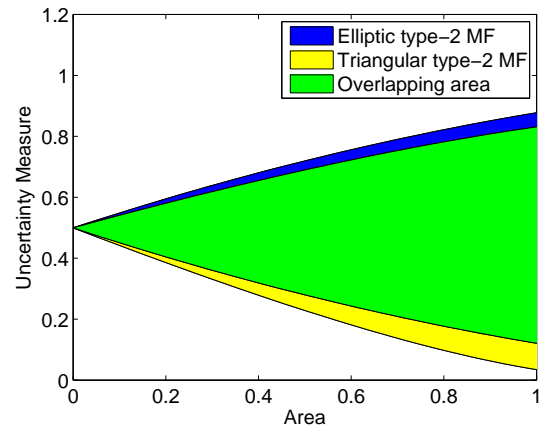

(c) Method proposed by Cornelis and (d) Method proposed based on MendelKerre.

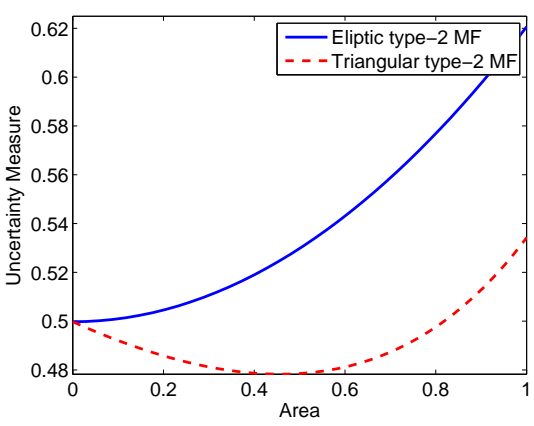

(b) Method proposed by Zeng and Li.

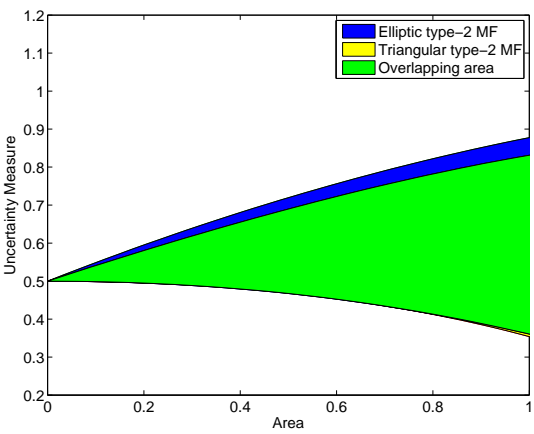

John Representation theorem.

Figure 5: Uncertainty measures using different methods.

\section{Arithmetic Using Different Type-2 Fuzzy MFs}

In this Section, we consider how uncertainty is propagated through two arithmetic operation: addition and multiplication.

\subsection{Addition}

It is well understood that addition can be performed on type- 1 fuzzy sets by applying addition to the parameters of the type-1 fuzzy sets. We now show that this is also the case for triangular, Gaussian and elliptic interval type-2 fuzzy sets.

The addition of two type-1 fuzzy sets $A$ and $B$ is given in (26) where values of $x, y$ and $z$ all belong to the same numeric domain. Since all operations on an interval type- 2 fuzzy can be defined by operations on the upper and lower MFs as 
defined by 27) and 28):

$$
\begin{aligned}
& \mu_{A+B}(z)=\underset{\forall z=x+y}{\operatorname{argmax}}\left(\mu_{A}(x) \wedge \mu_{B}(y)\right) \\
& \mu_{\underline{A \tilde{+} B}}(z)=\underset{\forall z=x+y}{\operatorname{argmax}}\left(\mu_{\underline{\tilde{A}}}(x) \wedge \mu_{\underline{\tilde{B}}}(y)\right) \\
& \mu_{\overline{A \tilde{+} B}}(z)=\underset{\forall z=x+y}{\operatorname{argmax}}\left(\mu_{\tilde{\tilde{A}}}(x) \wedge \mu_{\tilde{\tilde{B}}}(y)\right) .
\end{aligned}
$$

We now perform addition on three pairs of broadly equivalent triangular, Gaussian and elliptic interval type-2 fuzzy sets. Table 3 gives the parameters of the these set and the expected parameters of the sets resulting from the addition of these sets. These parameters are taken from the Authors previous work on addition of type-2 fuzzy sets [38, 39, 40].

Figures 6(a), 6(b) and 6(c) depict these addition operations of the respective three forms of MF; triangular, Gaussian and elliptic. The relationship between the parameters under addition is quite straightforward. To add a pair of triangular or Gaussian interval fuzzy numbers we simply add their respective parameters. This is a useful property which is well known for triangular and Gaussian fuzzy sets and here we will now show an equivalent property for elliptic fuzzy sets.

The parameters of an elliptic fuzzy set $\tilde{C}$ can be calculated from the two elliptic fuzzy sets $\tilde{A}$ and $\tilde{B}$ whose addition it results from. The following equations given this relation ship where $a_{1}, a_{2}, c$ and $c$ are set parameters and the subscripts denote which set the parameters belong to:

$$
\begin{gathered}
c_{\tilde{C}}=c_{\tilde{A}}+c_{\tilde{B}} \\
d_{\tilde{C}}=d_{\tilde{A}}+d_{\tilde{B}} \\
a 1_{\tilde{A}}=a 1_{\tilde{B}}=a 1_{\tilde{C}} \\
a 2_{\tilde{A}}=a 2_{\tilde{B}}=a 2_{\tilde{C}} .
\end{gathered}
$$

Table 1: Parameters of Sets Undergoing Addition.

\begin{tabular}{|c|c|c|c|c|c|c|c|c|c|}
\hline Type & \multicolumn{3}{|c|}{ About 3} & \multicolumn{3}{c|}{ About 12} & \multicolumn{3}{c|}{ About 15} \\
\hline Triangular $(\underline{s}, m, \underline{e})$ & 1.5 & 3 & 4.5 & 10.5 & 12 & 13.5 & 12 & 15 & 18 \\
\hline Triangular $(\bar{s}, m, \bar{e})$ & 1 & 3 & 5 & 10 & 12 & 14 & 11 & 15 & 19 \\
\hline Gaussian $(c, \underline{\sigma})$ & 3 & \multicolumn{2}{|c|}{0.5} & 12 & \multicolumn{2}{|c|}{0.5} & 15 & 1.0 \\
\hline Gaussian $(c, \bar{\sigma})$ & 3 & \multicolumn{2}{|c|}{0.8} & 12 & \multicolumn{2}{|c|}{0.8} & 15 & \multicolumn{2}{|c|}{1.6} \\
\hline Elliptic $\left(c, d, a_{1}\right)$ & 3 & 2 & 0.8 & 12 & 2 & 0.8 & 15 & 4 & 0.8 \\
\hline Elliptic $\left(c, d, a_{2}\right)$ & 3 & 2 & 1.2 & 12 & 2 & 1.2 & 15 & 4 & 1.2 \\
\hline
\end{tabular}




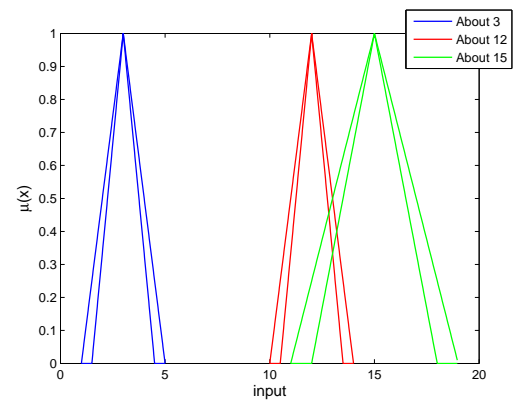

(a) Two triangular interval fuzzy sets.

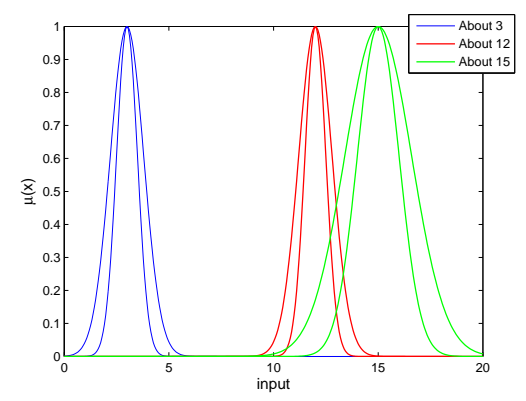

(b) Two Gaussian interval fuzzy sets.

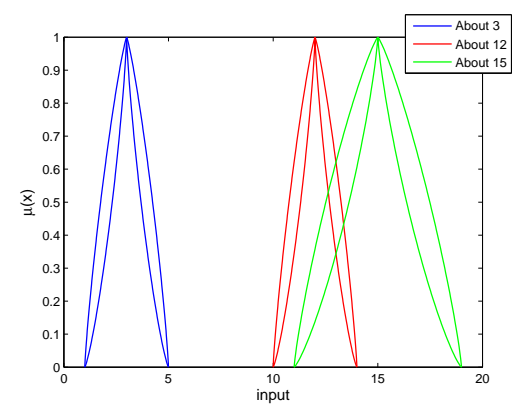

(c) Two Elliptic interval fuzzy sets.

Figure 6: The addition of different interval fuzzy sets About 3 and About 12.

\subsection{Multiplication}

Multiplication of fuzzy numbers of any kind is a more complex operation than addition. Typically, although not exclusively, multiplication is performed via $\alpha$ cuts as given by (33):

$$
C=\forall_{\alpha \in[0,1]} A_{\alpha} \times B_{\alpha}
$$

For continuous fuzzy sets, $A_{\alpha}$ and $B_{\alpha}$ are intervals and, therefore, interval arithmetic may be used. For discrete fuzzy sets, $A_{\alpha}$ and $B_{\alpha}$ are crisp sets, however, only minimum and maximum values in these sets need be multiplied. As with addition multiplication of type- 2 interval fuzzy sets can be performed on the upper and lower MFs independently as given in (34) and (35):

$$
\begin{aligned}
& \underline{C}=\forall_{\alpha \in[0,1]} \underline{A}_{\alpha} \times \underline{B}_{\alpha} \\
& \bar{C}=\forall_{\alpha \in[0,1]} \bar{A}_{\alpha} \times \bar{B}_{\alpha} .
\end{aligned}
$$




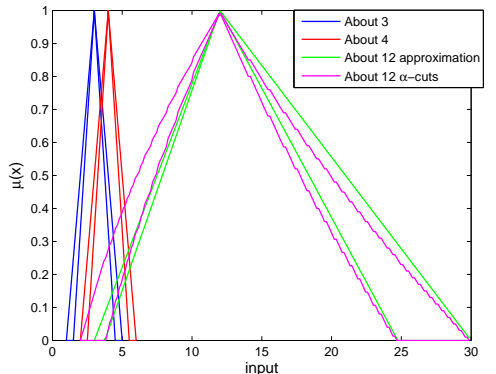

(a) Two triangular interval fuzzy sets.

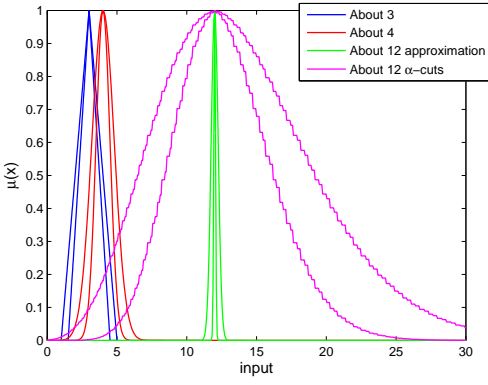

(b) Two Gaussian interval fuzzy sets.

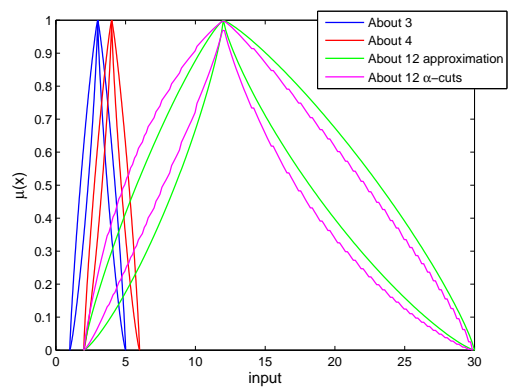

(c) Two Elliptic interval fuzzy sets.

Figure 7: The multiplication of different interval fuzzy sets About 3 and About 12.

We now perform multiplication on three pairs of broadly equivalent triangular, Gaussian and elliptic interval type- 2 fuzzy sets. Table 2 gives the parameters of the these sets. These parameters are taken from the Authors previous work on addition of type- 2 fuzzy sets [38, 39, 40].

Table 2: Parameters of Sets Undergoing Multiplication.

\begin{tabular}{|c|c|c|c|c|c|c|c|c|c|}
\hline Type & \multicolumn{3}{|c|}{ About 3} & \multicolumn{3}{c|}{ About 4} & \multicolumn{3}{c|}{ About 12} \\
\hline Triangular $(\underline{s}, m, \underline{e})$ & 1.5 & 3 & 4.5 & 2.5 & 4 & 5.5 & 3.75 & 12 & 24.75 \\
\hline Triangular $(\bar{s}, m, \bar{e})$ & 1 & 3 & 5 & 2 & 4 & 6 & 3 & 12 & 30 \\
\hline Gaussian $(c, \underline{\sigma})$ & 3 & 0.5 & 4 & 0.5 & 12 & \multicolumn{2}{|c|}{0.25} \\
\hline Gaussian $(c, \bar{\sigma})$ & 3 & 0.8 & 4 & \multicolumn{2}{|c|}{0.8} & 12 & \multicolumn{3}{c|}{0.64} \\
\hline Elliptic $\left(c, d, a_{1}\right)$ & 3 & 2 & 0.8 & 4 & 2 & 0.8 & 12 & See $(36)$ & 0.8 \\
\hline Elliptic $\left(c, d, a_{2}\right)$ & 3 & 2 & 1.2 & 4 & 2 & 1.2 & 12 & See $(\overline{36}$ & 1.2 \\
\hline
\end{tabular}


Figures 7(a), 7(b) and 7(c) depict these multiplication operations of the respective three forms of MF; triangular, Gaussian and elliptic using 10,000 $\alpha$-cuts. Unlike the addition, for multiplication of two fuzzy numbers does not yield a fuzzy set which can be easily constructed from its parameters. However, the parameters of the two sets being added can give a fuzzy set with a new set of parameters which approximate the result of multiplication.

For triangular and elliptic type-2 interval MFs the relevant parameters can be calculated. For triangular MFs all parameters are multiplied and this yields the set depicted in Fig. 7(a). For elliptic MFs the centres $(C)$ are multiplied together and the widths are given by 36 .

$$
d_{\tilde{C}}(x)=\left\{\begin{array}{ll}
c_{\tilde{C}}-\left(c_{\tilde{A}}-d_{\tilde{A}}\right) \times\left(c_{\tilde{B}}-d_{\tilde{B}}\right) & \text { if } x \leq c_{\tilde{C}} \\
\left(c_{\tilde{A}}-d_{\tilde{A}}\right) \times\left(c_{\tilde{B}}-d_{\tilde{B}}\right)-c_{\tilde{C}} & \text { if } x>c_{\tilde{C}}
\end{array} .\right.
$$

From Figs. 7(a), 7(b) and 7(c) it is clear that it is possible to approximate the multiplication operation quite easily with triangular and elliptic fuzzy set but not with Gaussian.

\section{Simulation Results}

Time series prediction is a well-known area of machine learning [41]. In this section, the prediction capability of IT2FLS with elliptic type-2 MFs for crude oil price dataset is studied. The prediction of oil price is very crucial as energy market has a great impact on other financial markets. The data set used belongs to the period from 02-Jan-1986 to 25-Apr-2016. The total number of samples in the experiment is equal to 7751 with $70 \%$ being used as the training samples and $30 \%$ is used as the test data. The data set is normalized to the interval of $[0,1]$ and converted to the true values after prediction. It is desired to show that the prediction performance of IT2FLSs with elliptic type-2 MFs is comparable and even may be better when compared to other more commonly used type-2 MFs.

Extreme learning machine (ELM) is a non-repetitive training method for singlehidden layer feedforward neural networks which does not include so many design parameters. The fact that this method does not include many design parameters makes it possible to get the best possible results all at once. This algorithm is used to train IT2FLS with single-hidden layer feedforward neural network structure [42].

An IF-THEN rule in IT2FLS in SLFN structure can be expressed as follows: 
$R^{n}: \operatorname{IF} x_{1}$ is $\tilde{X}_{1}^{n}$ and $x_{2}$ is $\tilde{X}_{2}^{n}$ and $\cdots$ and $x_{d}$ is $\tilde{X}_{d}^{n}$ THEN $y^{n}(\mathbf{x})=c_{0}^{n}+c_{1}^{n} x_{1}+\cdots+c_{d}^{n} x_{d}, \quad n=1, \cdots, N$, where $x_{i}$ 's stand for the inputs of the system, $R^{n}$ represents the $n^{\text {th }}$ rule of this structure, $N$ is the total number of rules which is taken as equal to 40 , the parameters $c_{i}^{j}$,s denote the consequent part parameters.

Since IT2FLSs with uncertain parameters in the consequent part are complex to deal with, in this section, A2-C0 fuzzy structure is preferred [43]. This type of MF benefits from interval type- 2 MFs in the antecedent part and crisp values for the consequent part and its output is calculated as follows.

$$
Y_{T S K / A 2-C 0}=\int_{w^{1} \in\left[\underline{w}^{1}, \bar{w}^{1}\right]} \ldots \int_{w^{M} \in\left[\underline{w}^{M}, \bar{w}^{M}\right]} 1 / \frac{\sum_{j=1}^{M} w^{j} F_{j}}{\sum_{j=1}^{M} w^{j}},
$$

where $\underline{w}^{j}$ and $\bar{w}^{j}$ are given by:

$$
\begin{gathered}
\underline{w}^{j}(x)=\underline{\mu}_{\tilde{F}_{1}^{j}}\left(x_{1}\right) \ldots \underline{\mu}_{\tilde{F}_{n}^{j}}\left(x_{n}\right) \\
\bar{w}^{j}(x)=\bar{\mu}_{\tilde{F}_{1}^{j}}\left(x_{1}\right) \ldots \bar{\mu}_{\tilde{F}_{n}^{j}}\left(x_{n}\right),
\end{gathered}
$$

with $F_{j}$ being defined as $F_{j}=\sum_{i=1}^{n} \alpha_{i j} x_{i}+\beta_{j}$ and $\underline{\mu}_{\tilde{F}_{k}^{j}}\left(x_{k}\right)$ are $\bar{\mu}_{\tilde{F}_{k}^{j}}\left(x_{k}\right)$ are the lower and the upper bounds of interval type- 2 membership function corresponding to $j^{\text {th }}$ rule for $x_{k}$. The MacLaurin based first order approximate output of the fuzzy system in closed form is obtained as follows:

$$
y=\frac{y_{r}+y_{l}}{2},
$$

where $y_{l}$ and $y_{r}$ are being the lower and upper bounds of the output of type-2 fuzzy system which are calculated as follows [44]:

$$
y_{r} \approx \frac{\sum_{j=1}^{M}\left(\bar{w}^{j}+\underline{w}^{j}\right) F^{j}+\sum_{j=1}^{M}\left(\operatorname{sign}\left(\bar{m}^{j}\right) \Delta w^{j} F^{j}\right)}{\sum_{j=1}^{M}\left(\bar{w}^{j}+\underline{w}^{j}\right)+\sum_{j=1}^{M}\left(\operatorname{sign}\left(\bar{m}^{j}\right) \Delta w^{j}\right)},
$$

where:

$$
\bar{m}^{j}=F^{j}-\frac{\sum_{j=1}^{M} \bar{w}^{j} F^{j}}{\sum_{j=1}^{M} \bar{w}^{j}}
$$


and $\Delta w^{j}=\bar{w}^{j}-\underline{w}^{j}$. Furthermore, $y_{l}$ is calculated as follows:

$$
y_{l} \approx \frac{\sum_{j=1}^{M}\left(\bar{w}^{j}+\underline{w}^{j}\right) F^{j}-\sum_{j=1}^{M}\left(\operatorname{sign}\left(\underline{m}^{j}\right) \Delta w^{j} F_{j}\right)}{\sum_{j=1}^{M}\left(\bar{w}^{j}+\underline{w}^{j}\right)-\sum_{j=1}^{M}\left(\operatorname{sign}\left(\underline{m}^{j}\right) \Delta w^{j}\right)},
$$

where:

$$
\underline{m}^{j}=F^{j}-\frac{\sum_{j=1}^{M} \bar{w}^{j} F^{j}}{\sum_{j=1}^{M} \bar{w}^{j}} .
$$

As Gaussian type-2 MFs with uncertain standard deviation parameter is one of the most frequently used type-2 MFs, this MF is used for demonstrate the power of elliptic type-2 MF in prediction. The parameters taken for this MF are defined as to be equal to $\bar{\sigma}=\sigma+\Delta$ and $\bar{\sigma}=\sigma-\Delta$.

The membership parameters taken for the elliptic type-2 MFs and Gaussian type$2 \mathrm{MF}$ with uncertain $\sigma$ values are depicted in Table 3 .

The results achieved are evaluated through the most commonly used errorbased measures, i.e., symmetric mean absolute percentage error (SMAPE), maximum error and root mean squared error (RMSE) [45]:

$$
\begin{gathered}
S M A P E \%=\frac{1}{N} \sum_{t=1}^{N} \frac{\left|A_{t}-F_{t}\right|}{\left(\left|F_{t}\right|+\left|A_{t}\right|\right) / 2} \times 100, \\
R M S E=\sqrt{\frac{1}{N} \sum_{t=1}^{N}\left(A_{t}-F_{t}\right)^{2},}
\end{gathered}
$$

where $N$ is the number of test samples, $A_{t}$ and $F_{t}$ are the actual and forecasted load for time $t$.

The mean value of root mean squared error (RMSE), the maximum value of error and SMAPE for IT2FLSs with both type-2 MFs namely Gaussian type-2 MF with uncertain $\sigma$ parameter and elliptic type-2 MF over 30 times of run of the programs are represented in Table 4. As can be seen from this table, the results obtained by elliptic type-2 MF shows significant improvements when compared to the results obtained by Gaussian type-2 MF with uncertain $\sigma$ parameter. The 


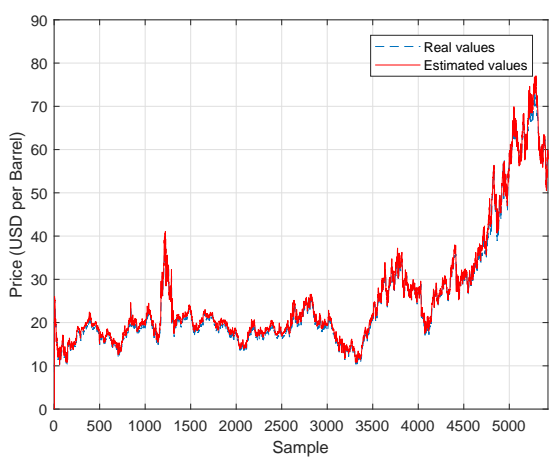

(a) Train data.

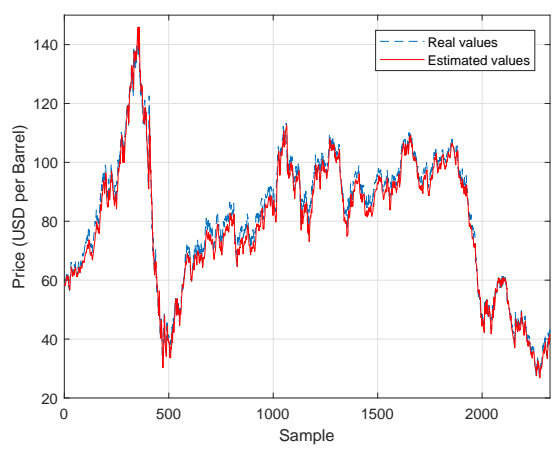

(c) Test data.

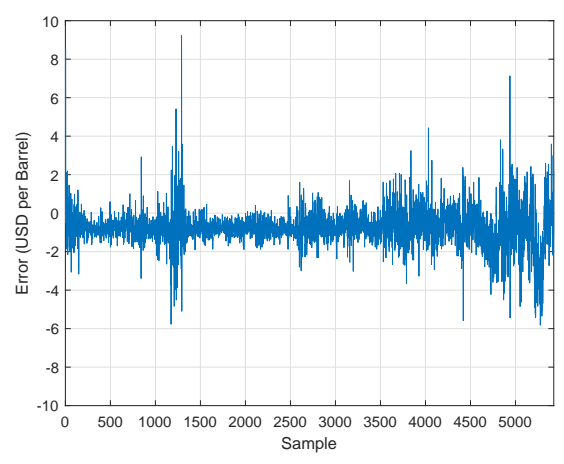

(b) Train data error.

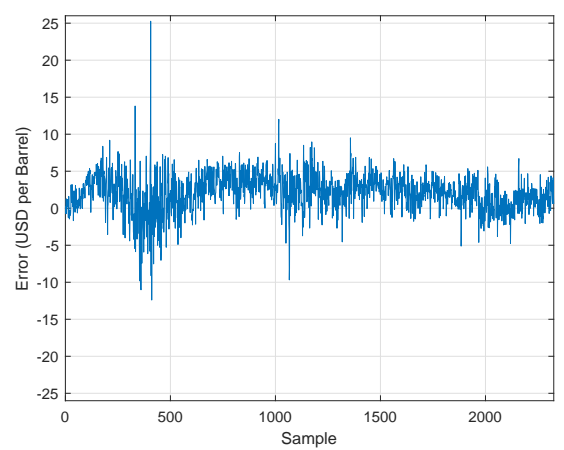

(d) Test data error.

Figure 8: Daily prediction results of crude oil price by using IT2FLS with elliptic type-2 MF.

prediction results for the train and test data are illustrated in Figs 8(a) and 8(c), respectively. As can be seen from figures, even though the test data seems to be very different from the train data, the prediction is obtained with a high performance.

Table 3: Parameters considered for type-2 MFs for the prediction of financial data set.

\begin{tabular}{|c|c|c|c|}
\hline \multicolumn{2}{|c|}{ Elliptic type-2 MF } & \multicolumn{2}{c|}{ Gaussian type-2 MF } \\
\hline Parameter & Interval & Parameter & Interval \\
\hline$c$ & {$[0,1]$} & $c$ & {$[0,1]$} \\
\hline$d$ & {$[0.95,1.3]$} & $\sigma$ & {$[0,1]$} \\
\hline$a_{1}$ & {$[1,1.1]$} & $\Delta$ & {$[0,0.05]$} \\
\hline$a_{2}$ & {$[0.9,1]$} & \multicolumn{2}{|c}{} \\
\cline { 1 - 2 } & \multicolumn{2}{|c}{} &
\end{tabular}


Table 4: Comparison results for the error obtained for test and train data sets.

\begin{tabular}{|l|c|c|c|c|c|c|}
\hline Type-2 MF used & \multicolumn{3}{|c|}{ Test } & \multicolumn{3}{c|}{ Train } \\
\hline & RMSE & Max & SMAPE & RMSE & Max & SMAPE \\
\hline Elliptic & 4.25 & 23.51 & 4.07 & 2.33 & 9.68 & 10.73 \\
\hline $\begin{array}{l}\text { Gaussian with } \\
\text { Uncertain } \sigma\end{array}$ & 7.30 & 30.58 & 6.92 & 4.31 & 11.62 & 21.91 \\
\hline
\end{tabular}

\section{Experimental Results}

The sector of unmanned aerial vehicles (UAVs) is the fastest growing sector of the aerospace industry. In particular, vertical take-off and landing (VTOL) UAVs have been the main interest of active research among the class of versatile flying robotic platforms due to their exceptional movement agility, relatively small size, and capability to hover and operate in cluttered environments. However, the design of the flight controller for multirotors is still a fundamental problem. Since quadrotor is an underactuated system with highly nonlinear dynamics. Thus, the controller design is not a straightforward task. Furthermore, in typical surveillance operations, the designed controller should provide precise trajectory tracking capabilities to enhance UAVs flight safety and task survivability. Hence, a robust and stable performance is required from designed controller regardless of unforeseen internal and external uncertainties.

\subsection{Mathematical model of the quadrotor UAV}

Let $\overrightarrow{\mathbf{F}}_{\mathcal{W}}=\left\{\overrightarrow{\mathbf{x}}_{\mathcal{W}}, \overrightarrow{\mathbf{y}}_{\mathcal{W}}, \overrightarrow{\mathbf{z}}_{\mathcal{W}}\right\}$ be the world fixed frame and $\overrightarrow{\mathbf{F}}_{\mathcal{B}}=\left\{\overrightarrow{\mathbf{x}}_{\mathcal{B}}, \overrightarrow{\mathbf{y}}_{\mathcal{B}}, \overrightarrow{\mathbf{z}}_{\mathcal{B}}\right\}$ be the body frame. The origin of the body frame is located at the center of mass (COM) of the quadrotor, as illustrated in Fig. 9. The absolute position of the quadrotor $\mathbf{p}=\left[\begin{array}{lll}x & y & z\end{array}\right]^{T}$ is described by the three Cartesian coordinates of its COM in $\overrightarrow{\mathbf{F}}_{\mathcal{W}}$. The time derivative of the position is given by $\mathbf{v}=$ $\left[\begin{array}{ccc}\dot{x} & \dot{y} & \dot{z}\end{array}\right]^{T}=\left[\begin{array}{lll}u & v & w\end{array}\right]^{T}$, where $\mathbf{v}$ is the absolute linear velocity of the quadrotor's COM expressed in $\overrightarrow{\mathbf{F}}_{\mathcal{W}}$. The attitude of the quadrotor $\mathbf{o}=\left[\begin{array}{lll}\phi & \theta & \psi\end{array}\right]^{T}$ is described by the three Euler's angles. The time derivative of the angles $\mathbf{o}$ is given by $\boldsymbol{\omega}=\left[\begin{array}{ccc}\dot{\phi} & \dot{\theta} & \dot{\psi}\end{array}\right]^{T}$ and the angular velocity expressed in $\overrightarrow{\mathbf{F}}_{\mathcal{B}}$ is $\boldsymbol{\omega}_{\mathcal{B}}=\left[\begin{array}{lll}p & q & r\end{array}\right]^{T}$. 


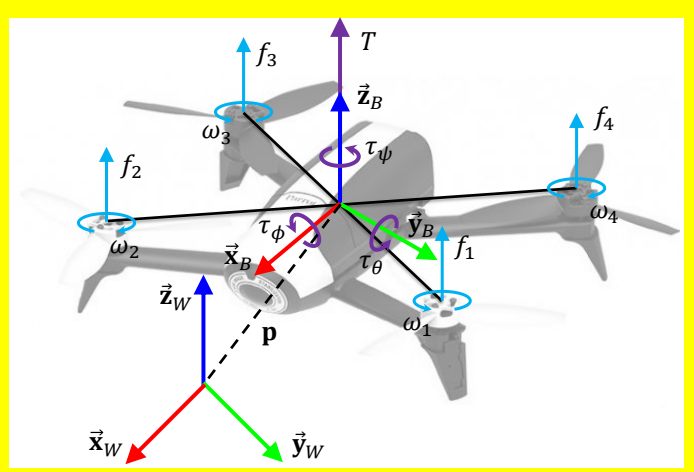

Figure 9: Quadrotor model with reference frames.

Using the definition of the velocity and the second Newton's law, the translational dynamic equations are as follows:

$$
\begin{cases}\dot{x}=u & \dot{u}=\frac{1}{m}(\cos \phi \cos \psi \sin \theta+\sin \phi \sin \psi) T \\ \dot{y}=v & \dot{v}=\frac{1}{m}(\cos \phi \sin \psi \sin \theta-\cos \psi \sin \phi) T \\ \dot{z}=w & \dot{w}=\frac{1}{m}(\cos \phi \cos \theta) T-g\end{cases}
$$

where $g$ is the gravitational acceleration, $m$ is the UAV's mass and $T$ is the total thrust. Using two Euler equation, the rotational dynamic equations are as follows:

$$
\begin{cases}\dot{\phi}=p+q \sin \phi \tan \theta+r \cos \phi \tan \theta & \dot{p}=\frac{I_{y}-I_{z}}{I_{x}} q r+\frac{1}{I_{x}} \tau_{\phi} \\ \dot{\theta}=q \cos \phi-r \sin \phi & \dot{q}=\frac{I_{z}-I_{x}}{I_{y}} p r+\frac{1}{I_{y}} \tau_{\theta} \\ \dot{\psi}=q \frac{\sin \phi}{\cos \theta}+r \frac{\cos \phi}{\cos \theta} & \dot{r}=\frac{I_{x}-I_{y}}{I_{z}} p q+\frac{1}{I_{z}} \tau_{\psi}\end{cases}
$$

where $\left(I_{x}, I_{y}, I_{z}\right)$ are moments of inertia and $\left(\tau_{\phi}, \tau_{\theta}, \tau_{\psi}\right)$ are three control torques. Finally, (46) and (47) provide the equations of the UAV's motion [46]. 


\subsection{Control scheme}

The overall architecture of the controller is illustrated in Fig. 10. It consists of two interconnected control loops: outer loop - position controller, and inner loop - velocity-attitude controller. The position controller is responsible for the quadrotor position tracking. While the velocity-attitude controller is responsible for the velocity tracking and the attitude stabilisation.

In this paper, three positional coordinates of the quadrotor are chosen as the controlled variables. Therefore, the trajectory generator provides the desired position $\mathbf{p}^{*}=\left[\begin{array}{lll}x^{*} & y^{*} & z^{*}\end{array}\right]^{T}$. The position controller consists of three independent controllers to track the desired position of the UAV along $x, y$ and $z$ axes. It takes

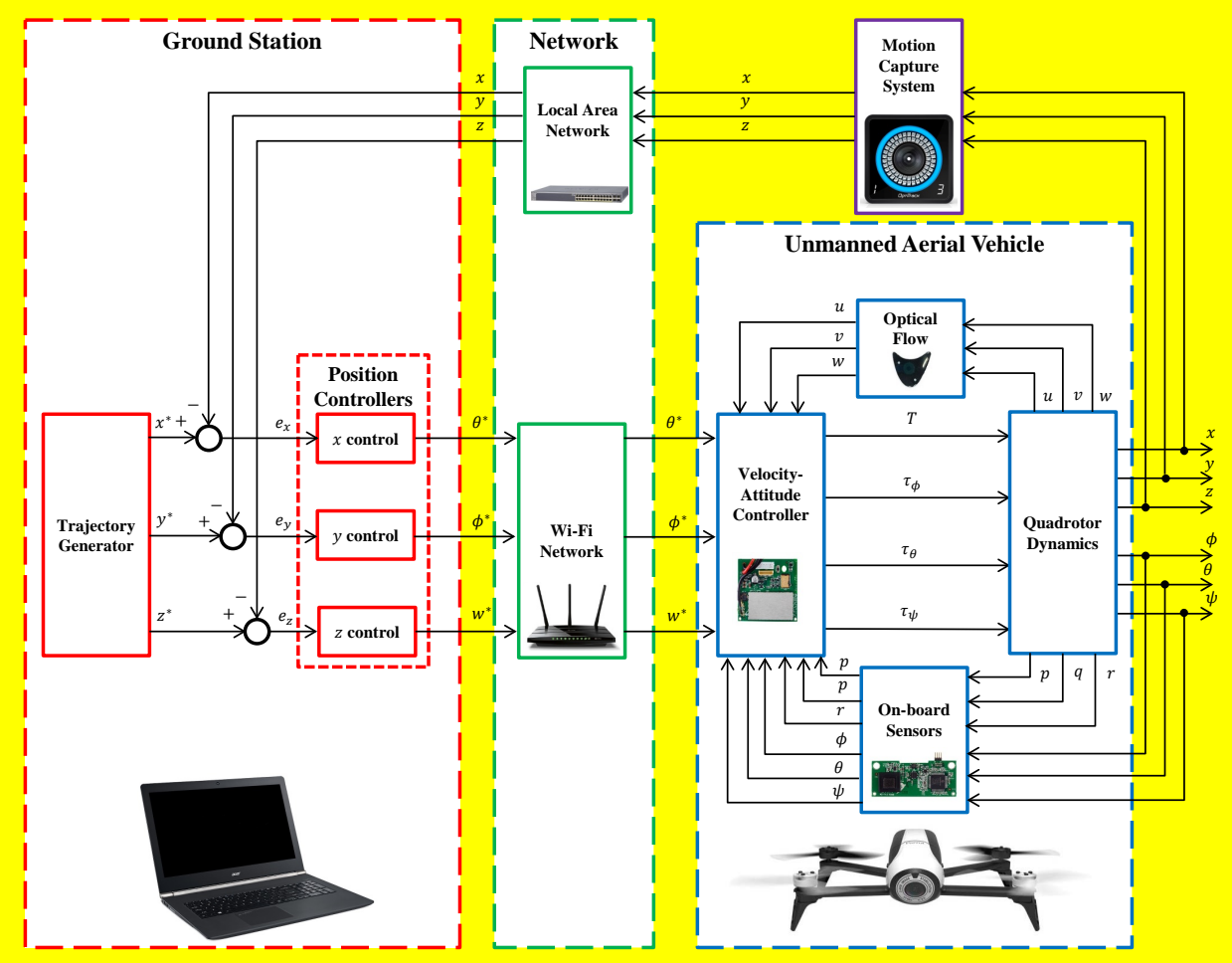

Figure 10: Block diagram of the control scheme for the quadrotor UAV. 
three position measurements and computes the position error:

$$
\mathbf{e}=\mathbf{p}^{*}-\mathbf{p}
$$

Then, it calculates the desired control inputs to the velocity-attitude controller in order to reach the desired position $\mathbf{p}^{*}$. The structure of the fuzzy position controller is presented in [47]. The fuzzy logic controller operates in parallel with conventional proportional-derivative (PD) controller. The PD controller is used to guarantee the stability of the system. The sliding mode control adaptation laws in [48] for the consequent parameters in fuzzy logic controllers are used. Another possibility is to use gradient descent based training algorithms as in [49].

\subsection{Experimental setup}

The experimental flight tests for the trajectory tracking problem were conducted in the indoor environment and evaluated in the Motion Capture Laboratory at Nanyang Technological University (NTU), Singapore. The laboratory environment is designed to use a set of eight OptiTrack Prime 13 cameras, to provide real-time pose (position and attitude) measurements of the UAV's COM with an update rate of $240 \mathrm{~Hz}$ and accuracy around $0.1 \mathrm{~mm}$. The pose data are routed to the controller trough the local network. The aircraft used for the experimental flight tests is Parrot Bebop 2 UAV, which is an attitude controlled commercial quadrotor. The Bebop Autonomy ROS package is used to communicate the output velocity from the controller to the quadrotor via a Wi-Fi connection.

\subsection{Trajectory Generation}

In the experimental scenario, a circle with radius $2 \mathrm{~m}$ is chosen for the trajectory tracking problem. In order to test the stability and robustness of the controllers, the reference speed is variable along the trajectory. The profile of the desired velocity is designed in a way to have different velocity conditions for each quarter of the circle, as shown in Fig. 11. In the first quarter $(t \in[0, \pi / 2))$, the speed gradually increases from $0 \mathrm{~m} / \mathrm{s}$ to $2 \mathrm{~m} / \mathrm{s}$. In the second quarter $(t \in[\pi / 2, \pi)$ ), the speed is kept constant at $2 \mathrm{~m} / \mathrm{s}$. In the third quarter $(t \in[\pi, 3 \pi / 2))$, the speed is increased again to reach $4 \mathrm{~m} / \mathrm{s}$. Finally, in the fourth quarter $(t \in[3 \pi / 2,2 \pi))$, the speed is decreased to $0 \mathrm{~m} / \mathrm{s}$. Then, the new circle starts and the velocity profile is repeated. 


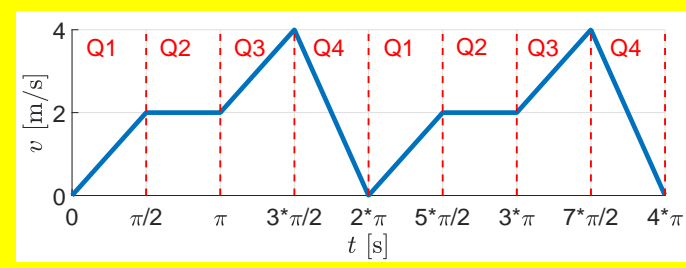

Figure 11: Velocity profile of the desired trajectory.

\subsection{Experimental performance}

The experimental results are evaluated through the most commonly used errorbased measures, i.e., root mean squared error (RMSE):

$$
R M S E=\sqrt{\frac{1}{N} \sum_{t=1}^{N}\left(p_{t}^{*}-p_{t}\right)^{2},}
$$

maximum absolute error:

$$
M A X=\max _{t \in N}\left|p_{t}^{*}-p_{t}\right|,
$$

and mean absolute error (MAE):

$$
M A E=\frac{1}{N} \sum_{t=1}^{N}\left|p_{t}^{*}-p_{t}\right|,
$$

where $N$ is the number of test samples, $p_{t}^{*}$ and $p_{t}$ are the actual and desired position at time $t$, respectively.

In Fig. 12, the trajectory tracking performance of interval type-2 fuzzy logic controller with elliptic MFs is compared with the performances of conventional PD controller, type- 1 fuzzy logic controllers with Gaussian and elliptic MFs, and Type-2 fuzzy logic controllers with Gaussian MFs. The projections of the trajectory on $x, y$ and $z$ axes of five complete circles are shown in Fig. 13. As can be seen, the steady state error is reduced because of learning capabilities of fuzzy logic controllers. This can also be seen from the Euclidean error and the average RMSE values from ten experiments which are shown in Fig. 14 and Table 8. In Fig. 15, the control signal generated by PD controller and type-2 fuzzy logic controller with elliptic MFs are shown. 


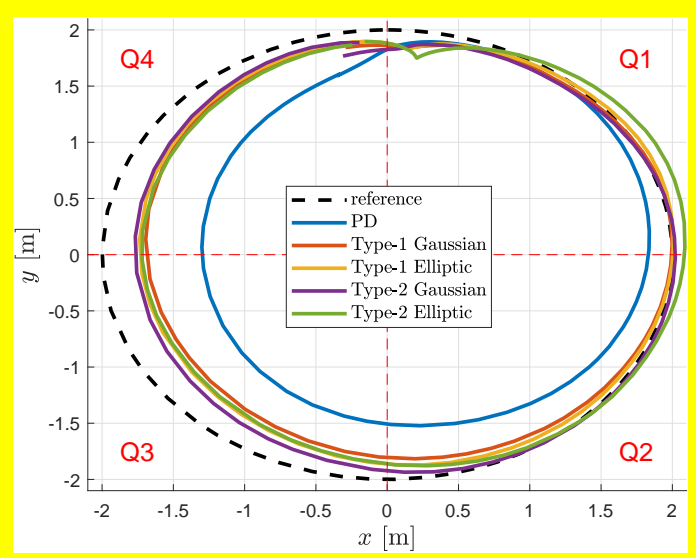

Figure 12: 3D trajectory tracking of different controllers.

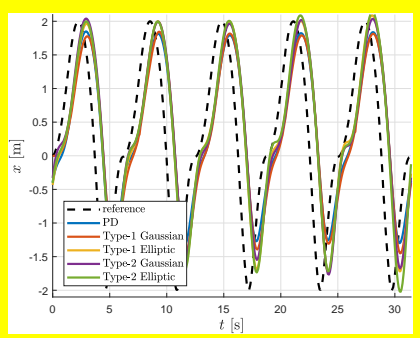

(a) $x$-axis tracking.

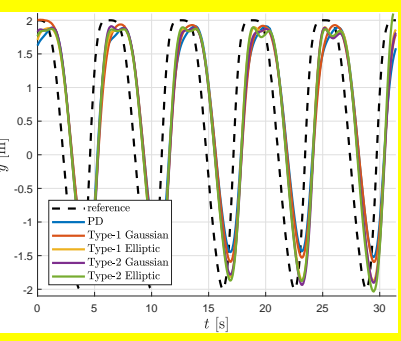

(b) $y$-axis tracking.

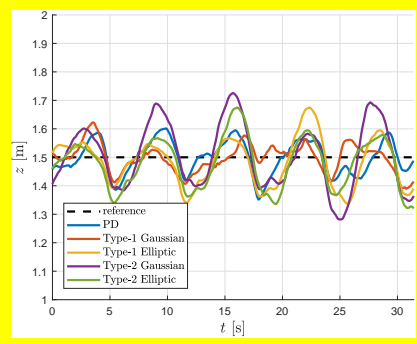

(c) $z$-axis tracking.

Figure 13: Trajectory tracking for $x, y$ and $z$ axes of different controllers.

\section{Conclusion}

\subsection{Conclusion}

There exist neither a systematic way to choose a fuzzy MF in order to achieve a better uncertainty modeling capability nor an objective criterion to check its performance. Another subjective analysis is made in this paper in which we have compared and contrasted the different ways of modeling uncertainty for different type-2 MFs in literature. Our findings say that having certain values on the center and the left and right end-points of the support are more interpretable when injecting the expert knowledge into the fuzzy system design. The time series prediction 


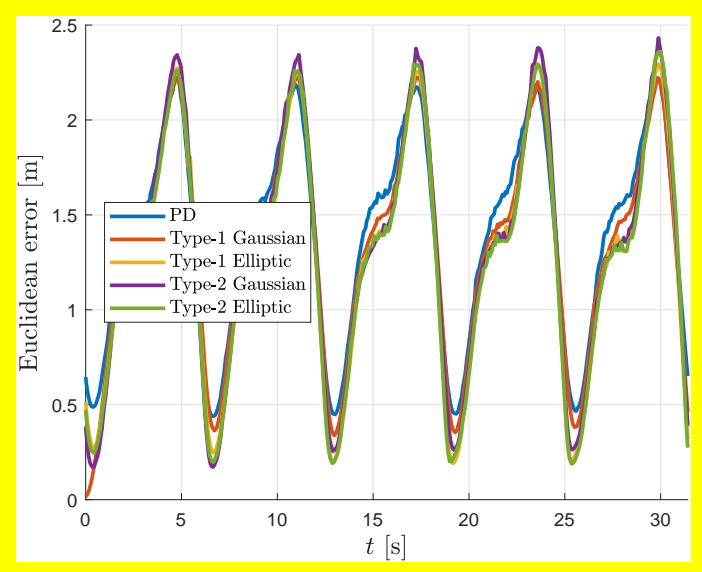

Figure 14: Euclidean error of different controllers.

Table 5: Comparison results for the error from different controllers.

\begin{tabular}{|l|c|c|c|}
\hline Controller & RMSE & Max Error & MAE \\
\hline PD & 1.595 & 2.224 & 1.490 \\
\hline Type-1 FNN with Gaussian MFs & 1.455 & 2.326 & 1.339 \\
\hline Type-1 FNN with elliptic MFs & 1.418 & 2.296 & 1.277 \\
\hline Type-2 FNN with Gaussian MFs & 1.445 & 2.432 & 1.309 \\
\hline Type-2 FNN with elliptic MFs & 1.416 & 2.361 & 1.270 \\
\hline
\end{tabular}

results show that elliptic MFs have comparable prediction results when compared to Gaussian and triangular MFs. Moreover, fuzzy logic controller with elliptic MFs gives similar results to fuzzy logic controller with Gaussian MFs. However, it has better performances when compared to conventional PD controller. On the other hand, they have a number of advantages when compared to the conventional MFs. We believe that the presented results will encourage researchers to use elliptic MFs in real-world identification and control applications as the proposed MF is easy to interpret in addition to its unique features.

\subsection{Future work}

Further work will consider the role of type-2 fuzzy elliptic MFs in real applications modelling human decision making. Unlike time series prediction and 


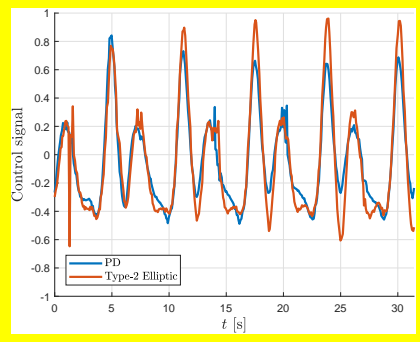

(a) $x$-axis control signal.

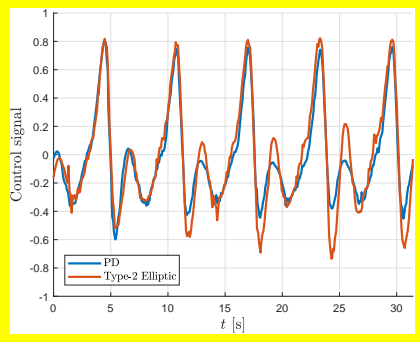

(b) $y$-axis control signal.

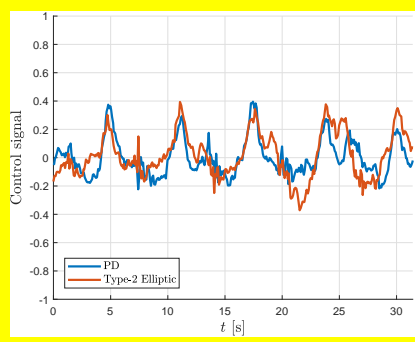

(c) $z$-axis control signal.

Figure 15: Control signal for $x, y$ and $z$ axes of PD and Type-2 Elliptic controllers.

control problems, the selection of MFs play a vital role in decision-making problems. Therefore, a more interpretable type- $2 \mathrm{MF}$ for such applications will be considered for modeling these systems.

\section{References}

[1] L. Zadeh, Fuzzy sets, Information and Control 8 (1965) 338-353.

[2] L. Zadeh, The concept of a linguistic variable and its application to approximate reasoning-I, Information Sciences 8 (3) (1975) 199 - 249. doi:http://dx.doi.org/10.1016/0020-0255(75) 90036-5.

URL http://www.sciencedirect.com/science/article/ pii/0020025575900365

[3] N. N. Karnik, J. M. Mendel, Q. Liang, Type-2 fuzzy logic systems, IEEE Trans. Fuzzy Syst. 7 (1999) 643-658.

[4] J. M. Mendel, R. John, F. Liu, Interval type-2 fuzzy logic systems made simple, IEEE Trans. Fuzzy Syst. 14 (2006) 808-821.

[5] J. Mendel, General type-2 fuzzy logic systems made simple: A tutorial, Fuzzy Systems, IEEE Transactions on 22 (5) (2014) 1162-1182. doi: $10.1109 /$ TFUZZ.2013.2286414.

[6] A. M. El-Nagar, M. El-Bardini, Parallel realization for self-tuning interval type-2 fuzzy controller, Engineering Applications of Artificial Intelligence 61 (2017) 8 - 20. doi:http://dx.doi.org/10.1016/j. 
engappai.2017.02.011.

URL http://www.sciencedirect.com/science/article/ pii/S0952197617300349

[7] B. E. Demir, R. Bayir, F. Duran, Real-time trajectory tracking of an unmanned aerial vehicle using a self-tuning fuzzy proportional integral derivative controller, International Journal of Micro Air Vehicles 8 (4) (2016) 252268.

[8] E. Kayacan, R. Maslim, Type-2 fuzzy logic trajectory tracking control of quadrotor vtol aircraft with elliptic membership functions, IEEE/ASME Transactions on Mechatronics 22 (1) (2017) 339-348.

[9] E. Camci, E. Kayacan, Game of drones: Uav pursuit-evasion game with type-2 fuzzy logic controllers tuned by reinforcement learning, in: 2016 IEEE International Conference on Fuzzy Systems (FUZZ-IEEE), 2016, pp. 618-625. doi:10.1109/FUZZ-IEEE.2016.7737744.

[10] M. Mehndiratta, E. Kayacan, T. Kumbasar, Design and experimental validation of single input type-2 fuzzy pid controllers as applied to 3 dof helicopter testbed, in: 2016 IEEE International Conference on Fuzzy Systems (FUZZ-IEEE), 2016, pp. 1584-1591. doi :10 .1109/FUZZ-IEEE. 2016.7737879 .

[11] C.-F. Juang, C.-H. Hsu, Reinforcement interval type-2 fuzzy controller design by online rule generation and q-value-aided ant colony optimization, Systems, Man, and Cybernetics, Part B: Cybernetics, IEEE Transactions on 39 (6) (2009) $1528-1542$.

[12] C. Juang, Y. Tsao, A self-evolving interval type-2 fuzzy neural network with online structure and parameter learning, IEEE Trans. on Fuzzy Systems 16 (2008) 1411-1424.

[13] R. Sepulveda, P. Melin, A. Rodriguez, A. Mancilla, O. Montiel, Analyzing the effects of the footprint of uncertainty in type-2 fuzzy logic controllers, Engineering Letters 13 (2006) 138-147.

[14] A. Sarabakha, C. Fu, E. Kayacan, T. Kumbasar, Type-2 Fuzzy Logic Controllers Made Even Simpler: From Design to Deployment for UAVs, IEEE Transactions on Industrial Electronics PP (99) (2017) 1-1. doi: $10.1109 /$ TIE.2017.2767546. 
[15] J. M. Mendel, Type-2 fuzzy sets and systems: a retrospective, Informatik Spektrum 38 (6) (2015) 523-532. doi:10.1007/ s00287-015-0927-4. URL http://dx.doi.org/10.1007/s00287-015-0927-4

[16] M. Khanesar, E. Kayacan, M. Teshnehlab, O. Kaynak, Analysis of the noise reduction property of type-2 fuzzy logic systems using a novel type- 2 membership function, Systems, Man, and Cybernetics, Part B: Cybernetics, IEEE Transactions on 41 (5) (2011) 1395-1406.

[17] E. Kayacan, S. Coupland, R. John, M. A. Khanesar, Elliptic membership functions and the modeling uncertainty in type-2 fuzzy logic systems as applied to time series prediction, in: 2017 IEEE International Conference on Fuzzy Systems (FUZZ-IEEE), 2017, pp. 1-7.

[18] J. Dombi, Membership function as an evaluation, Fuzzy Sets and Systems 35 (1) (1990) 1 - 21. doi:http://dx.doi.org/10.1016/ 0165-0114(90)90014-W.

URL http://www.sciencedirect.com/science/article/ pii/016501149090014W

[19] S. Medasani, J. Kim, R. Krishnapuram, An overview of membership function generation techniques for pattern recognition, International Journal of Approximate Reasoning 19 (3) (1998) $391-417$. doi:http: //dx.doi.org/10.1016/s0888-613x(98)10017-8.

URL http://www.sciencedirect.com/science/article/ pii/S0888613X98100178

[20] T. Ozen, J. M. Garibaldi, Effect of type-2 fuzzy membership function shape on modelling variation in human decision making, in: 2004 IEEE International Conference on Fuzzy Systems (IEEE Cat. No.04CH37542), Vol. 2, 2004, pp. 971-976 vol.2. doi:10.1109/FUZZY.2004.1375539.

[21] P. K. Muhuri, A. K. Shukla, Semi-elliptic membership function: Representation, generation, operations, defuzzification, ranking and its application to the real-time task scheduling problem, Engineering Applications of Artificial Intelligence 60 (2017) $71-82$. doi:http: //dx.doi.org/10.1016/j.engappai.2016.12.020.

URL http://www.sciencedirect.com/science/article/ pii/S0952197616302548 
[22] M. Khanesar, E. Kayacan, M. Reyhanoglu, O. Kaynak, Feedback error learning control of magnetic satellites using type-2 fuzzy neural networks with elliptic membership functions, Cybernetics, IEEE Transactions on 45 (4) (2015) 858-868. doi:10.1109/TCYB.2015.2388758.

[23] J. Rickard, J. Aisbett, G. Gibbon, Fuzzy Subsethood for Fuzzy Sets of Type2 and Generalized Type-n, Fuzzy Systems, IEEE Transactions on 17 (1) (2009) 50-60. doi:10.1109/TFUZZ.2008.2006369.

[24] J. Aisbett, J. Rickard, D. Morgenthaler, Type-2 fuzzy sets as functions on spaces, Fuzzy Systems, IEEE Transactions on 18 (4) (2010) 841-844. do i : $10.1109 /$ TFUZZ.2010.2046176.

[25] F. Olivas, F. Valdez, O. Castillo, A comparative study of membership functions for an interval type-2 fuzzy system used for dynamic parameter adaptation in particle swarm optimization, in: Recent Developments and New Direction in Soft-Computing Foundations and Applications, Springer, 2016, pp. 373-385.

[26] S. Medasani, J. Kim, R. Krishnapuram, An overview of membership function generation techniques for pattern recognition, International Journal of Approximate Reasoning 19 (34) (1998) 391 - 417. doi:http: //dx.doi.org/10.1016/S0888-613X(98)10017-8.

URL http://www.sciencedirect.com/science/article/ pii/s0888613X98100178

[27] $\mathrm{D}$. Wu, Twelve considerations in choosing between gaussian and trapezoidal membership functions in interval type-2 fuzzy logic controllers, in: Fuzzy Systems (FUZZ-IEEE), 2012 IEEE International Conference on, 2012, pp. 1-8. doi:10.1109/FUZZ-IEEE.2012.6251210.

[28] K. Ang, C. Quek, Supervised pseudo self-evolving cerebellar algorithm for generating fuzzy membership functions, Expert Systems with Applications 39 (3) (2012) 2279 - 2287. doi:http: //dx.doi.org/10.1016/j.eswa.2011.08.001.

URL http://www.sciencedirect.com/science/article/ pii/s0957417411011122

[29] M. Khanesar, E. Kayacan, M. Teshnehlab, O. Kaynak, Levenberg marquardt algorithm for the training of type-2 fuzzy neuro systems with a novel type-2 
fuzzy membership function, in: Advances in Type-2 Fuzzy Logic Systems (T2FUZZ), 2011 IEEE Symposium on, 2011, pp. 88-93. doi:10.1109/ T2FUZZ.2011.5949558.

[30] M. Khanesar, M. Teshnehlab, E. Kayacan, O. Kaynak, A novel type-2 fuzzy membership function: application to the prediction of noisy data, in: Computational Intelligence for Measurement Systems and Applications (CIMSA), 2010 IEEE International Conference on, 2010, pp. 128-133. doi:10.1109/CIMSA.2010.5611774.

[31] L.-X. Wang, A Course in Fuzzy Systems and Control, Prentice-Hall press, USA, 1997.

[32] D. Wu, J. M. Mendel, Uncertainty measures for interval type-2 fuzzy sets, Information Sciences 177 (23) (2007) 5378-5393.

[33] E. Szmidt, J. Kacprzyk, Entropy for intuitionistic fuzzy sets, Fuzzy sets and systems 118 (3) (2001) 467-477.

[34] W. Zeng, H. Li, Relationship between similarity measure and entropy of interval valued fuzzy sets, Fuzzy Sets and Systems 157 (11) (2006) 14771484.

[35] C. Cornelis, E. Kerre, Inclusion measures in intuitionistic fuzzy set theory, in: Symbolic and Quantitative Approaches to Reasoning with Uncertainty, Springer, 2003, pp. 345-356.

[36] J. M. Mendel, R. I. B. John, Type-2 fuzzy sets made simple, Fuzzy Systems, IEEE Transactions on 10 (2) (2002) 117-127.

[37] R. R. Yager, A measurement-informational discussion of fuzzy union and intersection, International Journal of Man-Machine Studies 11 (2) (1979) 189-200.

[38] S. Coupland, R. John, An Approach to Type-2 Fuzzy Arithmetic, in: Proc. UK Workshop on Computational Intelligence, 2003, pp. 107 - 114.

[39] W. Blewitt, S.-M. Zhou, S. Coupland, Type-2 fuzzy arithmetic, in: Proc. FUZZ-IEEE 2007, London, UK, 2007, pp. 1456 - 1461. 
[40] H. Hamrawi, S. Coupland, Type-2 fuzzy arithmetic using alpha-planes, in: Proceedings of IFSA/EUSFLAT 2009, Lisbon, Portugal, 2009, pp. 606 611.

[41] A. S. Weigend, N. A. Gershenfeld, Time series prediction: forecasting the future and understanding the past, Addison-Wesley Pub. Co.,, 1992.

[42] Z. Deng, K.-S. Choi, L. Cao, S. Wang, T2fela: Type-2 fuzzy extreme learning algorithm for fast training of interval type-2 tsk fuzzy logic system, Neural Networks and Learning Systems, IEEE Transactions on 25 (4) (2014) 664-676.

[43] J. M. Mendel, Uncertain Rule-Based Fuzzy Logic Systems: Introduction and New Directions, Prentice-Hall PTR, 2001.

[44] M. A. Khanesar, J. M. Mendel, Maclaurin series expansion complexityreduced center of sets type-reduction+ defuzzification for interval type-2 fuzzy systems, in: Fuzzy Systems (FUZZ-IEEE), 2016 IEEE International Conference on, IEEE, 2016, pp. 1224-1231.

[45] P. Mandal, T. Senjyu, N. Urasaki, T. Funabashi, A. Srivastava, A novel approach to forecast electricity price for pjm using neural network and similar days method, Power Systems, IEEE Transactions on 22 (4) (2007) 20582065. doi:10.1109/TPWRS.2007.907386.

[46] A. Sarabakha, E. Kayacan, Y6 Tricopter Autonomous Evacuation in an Indoor Environment using Q-learning Algorithm, in: 2016 IEEE 55th Conference on Decision and Control (CDC), 2016, pp. 5992-5997.

[47] A. Sarabakha, N. Imanberdiyev, E. Kayacan, M. A. Khanesar, H. Hagras, Novel LevenbergMarquardt based learning algorithm for unmanned aerial vehicles, Information Sciences 417 (2017) $361-380$. doi : https : //doi.org/10.1016/j.ins.2017.07.020.

URL https://www.sciencedirect.com/science/article/ pii/s0020025517308393

[48] E. Kayacan, M. A. Khanesar, Chapter 7 - Sliding Mode Control TheoryBased Parameter Adaptation Rules for Fuzzy Neural Networks, in: E. Kayacan, M. A. Khanesar (Eds.), Fuzzy Neural Networks for Real Time Control Applications, Butterworth-Heinemann, 2016, pp. 85 - 131. doi:https: 
//doi.org/10.1016/B978-0-12-802687-8.00007-4.

URL https://www.sciencedirect.com/science/article/ pii/B9780128026878000074

[49] E. Kayacan, O. Kaynak, R. Abiyev, J. Trresen, M. Hvin, K. Glette, Design of an adaptive interval type-2 fuzzy logic controller for the position control of a servo system with an intelligent sensor, in: International Conference on Fuzzy Systems, 2010, pp. 1-8. doi:10.1109/FUZZY.2010. 5584629 . 